\title{
Neural Communication Patterns Underlying Conflict Detection, Resolution, and Adaptation
}

\author{
Carina R. Oehrn, ${ }^{1}$ Simon Hanslmayr, ${ }^{2,3}$ Juergen Fell, ${ }^{1}$ Lorena Deuker, ${ }^{1,4}$ Nico A. Kremers, ${ }^{1}$ Anne T. Do Lam, \\ Christian E. Elger, ${ }^{1}$ and Nikolai Axmacher ${ }^{1,5}$ \\ ${ }^{1}$ Department of Epileptology, University of Bonn, D-53105 Bonn, Germany, ${ }^{2}$ Department of Psychology-Zukunftskolleg, D-78457 Konstanz, Germany, \\ ${ }^{3}$ School of Psychology, University of Birmingham, Birmingham, UK-B15 2TT, ${ }^{4}$ Centre for Cognitive Neuroimaging, Donders Institute for Brain, Cognition \\ and Behaviour, Radboud University, 6525 EN Nijmegen, The Netherlands, and ${ }^{5}$ German Center for Neurodegenerative Diseases, D-53175 Bonn, Germany
}

In an ever-changing environment, selecting appropriate responses in conflicting situations is essential for biological survival and social success and requires cognitive control, which is mediated by dorsomedial prefrontal cortex (DMPFC) and dorsolateral prefrontal cortex (DLPFC). How these brain regions communicate during conflict processing (detection, resolution, and adaptation), however, is still unknown. The Stroop task provides a well-established paradigm to investigate the cognitive mechanisms mediating such response conflict. Here, we explore the oscillatory patterns within and between the DMPFC and DLPFC in human epilepsy patients with intracranial EEG electrodes during an auditory Stroop experiment. Data from the DLPFC were obtained from 12 patients. Thereof four patients had additional DMPFC electrodes available for interaction analyses. Our results show that an early $\theta(4-8 \mathrm{~Hz})$ modulated enhancement of DLPFC $\gamma$-band $(30-100 \mathrm{~Hz})$ activity constituted a prerequisite for later successful conflict processing. Subsequent conflict detection was reflected in a DMPFC $\theta$ power increase that causally entrained DLPFC $\theta$ activity (DMPFC to DLPFC). Conflict resolution was thereafter completed by coupling of DLPFC $\gamma$ power to DMPFC $\theta$ oscillations. Finally, conflict adaptation was related to increased postresponse DLPFC $\gamma$-band activity and to $\theta$ coupling in the reverse direction (DLPFC to DMPFC). These results draw a detailed picture on how two regions in the prefrontal cortex communicate to resolve cognitive conflicts. In conclusion, our data show that conflict detection, control, and adaptation are supported by a sequence of processes that use the interplay of $\theta$ and $\gamma$ oscillations within and between DMPFC and DLPFC.

Key words: dorsolateral prefrontal cortex; dorsomedial prefrontal cortex; $\gamma$; oscillations; Stroop; $\theta$

\section{Introduction}

Biological survival and social success depend crucially on overcoming predominant response tendencies that interfere with current goal-relevant responses (i.e., on the resolution of response conflicts). This important cognitive process is frequently investigated using the Stroop paradigm, in which participants indicate the ink color of a color word (e.g., "blue" written in red font; Stroop, 1935; for a review, see MacLeod, 1991). A conflict is elicited when the two stimulus dimensions are inconsistent and when the more automatic process (word reading) interferes with the current task (naming of the ink color). Three subprocesses

\footnotetext{
Received July 19, 2013; revised June 3, 2014; accepted June 22, 2014.

Author contributions: J.F., C.E.E., and N.A. designed research; C.R.O. and A.T.D.L. performed research; L.D. and N.A.K. contributed unpublished reagents/analytic tools; C.R.O., S.H., J.F., and N.A. analyzed data; C.R.O., S.H., J.F., and N.A. wrote the paper.

C.R.0. was supported by Deutsche Forschungsgemeinschaft Grant FE 366/5-1. L.D. was supported by Deutsche Forschungsgemeinschaft Grant AX82/1. N.A.K. was supported by a BONFOR dissertation stipend. S.H. and N.A. were supported by Deutsche Forschungsgemeinschaft through Emmy-Noether Grants HA5622/1-1 and AX82/2. We thank Tobias Staudigl, Marcin Leszczynski, and Amirhossein Jahanbekam for help with implementing Granger causality analyses.

The authors declare no competing financial interests.

Correspondence should be addressed to Dr. Nikolai Axmacher, Department of Epileptology, University of Bonn, Sigmund-Freud-Strasse 25, D-53105 Bonn, Germany. E-mail: nikolai.axmacher@ukb.uni-bonn.de.

DOI:10.1523/JNEUROSCI.3099-13.2014

Copyright $\odot 2014$ the authors $\quad 0270-6474 / 14 / 3410438-15 \$ 15.00 / 0$
}

have been suggested to be part of conflict processing (Botvinick et al., 2001; Mansouri et al., 2009): conflict detection, conflict resolution (i.e., suppression of a competing response in favor of an appropriate reaction), and adaptation (i.e., preparation for a conflict after an immediately preceding conflict).

Several fMRI studies revealed that brain regions within dorsomedial prefrontal cortex (DMPFC) and dorsolateral prefrontal cortex (DLPFC) play a crucial role in conflict processing (MacDonald et al., 2000; Botvinick et al., 2001, 2004; Kerns et al., 2004; Ridderinkhof et al., 2004; van Veen and Carter, 2005). Furthermore, previous EEG studies associated increases in DMPFC $\theta$ $(4-8 \mathrm{~Hz})$ power and DMPFC-DLPFC $\theta$ phase synchronization with conflict detection, response monitoring, and cognitive control (Trujillo and Allen, 2007; Cohen et al., 2008; Hanslmayr et al., 2008; Cavanagh et al., 2009, 2012; Womelsdorf et al., 2010). Similar results were obtained in 2 patients with laminar microelectrodes recorded from within the anterior cingulate cortex (Wang et al., 2005). In general, brain oscillations may support neural communication by synchronously opening and closing the time windows for neural firing (Varela et al., 2001; Fries, 2005, Fell and Axmacher, 2011; Siegel et al., 2012). On the other hand, several studies reported that activation of the DLPFC during executive control is associated with activity in the $\gamma$ frequency range (e.g., Jensen et al., 2007; Michels et al., 2010; Koga et al., 


\section{A DMPFC electrodes}
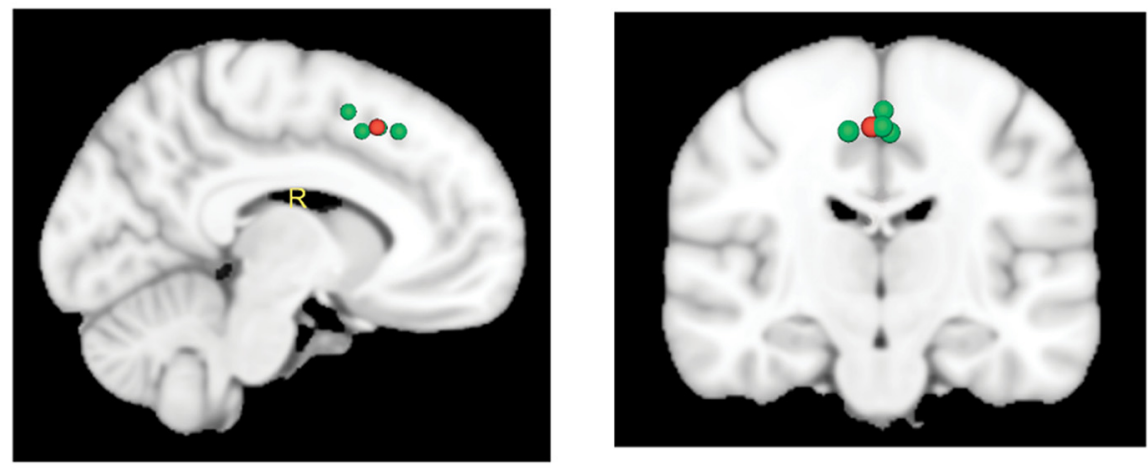

B DLPFC electrodes
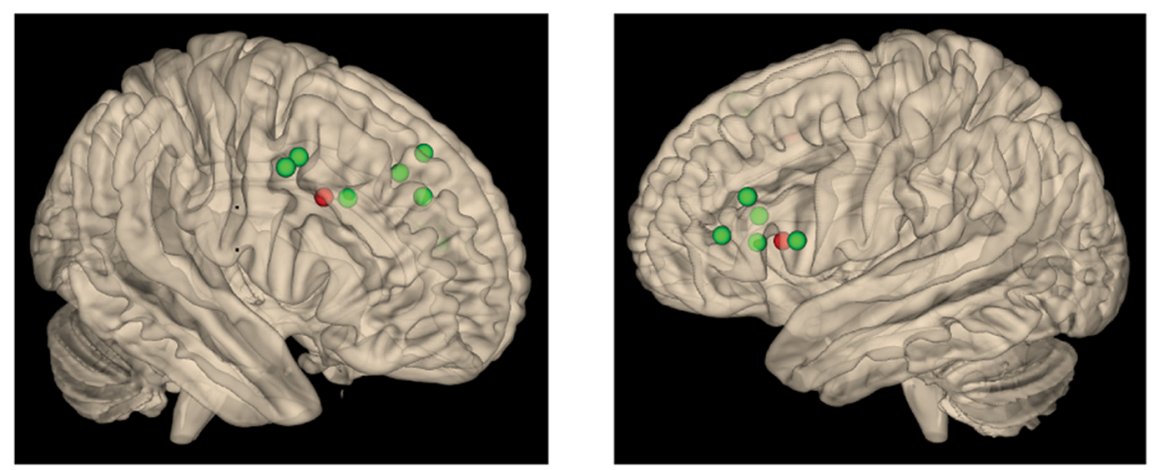

Figure 1. Electrode locations mapped onto MNI templates. $A$, DMPFC electrodes $(n=4)$. One contact per patient was chosen based on the minimal distance to a reference coordinate within the DMPFC, where conflict-associated changes in BOLD signal had been previously found in an fMRI experiment using an identical paradigm (Haupt et al., 2009) (in the contrast of conflict vs nonconflict trials in the phonetic condition; MNI coordinates: $2,18,46)$. Green represents the selected contact for each patient. Red represents the reference coordinate. $\boldsymbol{B}, \mathrm{DLPFC}$ electrodes $(n=12$; note that one patient did not have a preimplantation MRI which is necessary to determine MNI coordinates). Electrodes were selected correspondingly, with bilateral reference points in the DLPFC based on previous fMRI results (in the contrast of conflict vs nonconflict trials in the phonetic condition; MNI coordinates: 40, 16, 32 and $-48,18,10)$.

2011, Swann et al., 2013) ( 30-100 Hz). It has been suggested that $\gamma$ activity reflects local neuronal processes, whereas synchronized $\theta$ oscillations support long-range interactions (von Stein and Sarnthein, 2000; Buzsáki and Draguhn, 2004; Buzsáki, 2006). Furthermore, cross-frequency coupling (CFC) between $\theta$ phase and $\gamma$ amplitude appears to be important for the integration of local neuronal activity into large-scale cerebral networks (Ward, 2003; Lisman, 2005; Canolty et al., 2006; Jensen and Colgin, 2007; Axmacher et al., 2010; Canolty and Knight, 2010).

It is still an open question how DMPFC $\theta$ oscillations and DLPFC $\gamma$ activity interact and how they contribute to the three different subprocesses of conflict processing: conflict detection, resolution, and adaptation (Mansouri et al., 2009). Furthermore, the direction of information flow between DMPFC and DLPFC is still unclear (Botvinick et al., 2001; Morishima et al., 2010). Here, we addressed these issues by recording intracranial EEG from the DMPFC and DLPFC of 12 epilepsy patients during an auditory version of the Stroop paradigm (Haupt et al., 2009) and by analyzing the time-frequency pattern within these regions and between them.

\section{Materials and Methods}

Patients. We recorded intracranial EEG from 14 patients with pharmacoresistant epilepsy who had been implanted with intracranial electrodes for diagnostic purposes. Depending on the suspected ictal onset zone, patients had been implanted with frontal subdural strip and/or grid elec- trodes, enabling us to record electrophysiological activity from the DLPFC, and some with additional interhemispheric strip electrodes covering the DMPFC. In one patient, invasive diagnostics revealed seizure onset in the cingulate cortex and pathological activity in frontal electrodes. This patient was therefore excluded from further analysis. Furthermore, one patient was cognitively unable to follow instructions and was therefore excluded from analysis. In all remaining patients, exclusively data recorded contralateral to the epileptic focus were considered for analysis, where bilateral implantations were made. If only unilateral recordings were available, analyses were restricted to brain regions free of any seizurerelated activity or morphological changes in the MRI. This resulted in a final number of 12 patients ( 7 female; mean age \pm SD: $34 \pm 14$ years) with DLPFC ( 5 left, 7 right) electrodes included in the analysis, of which 4 had been additionally implanted with interhemispheric electrodes ( 3 left, 1 right). In 6 of these 12 patients, epileptic activity originated from medial temporal regions ( 2 contralateral, 4 ipsilateral), in 4 patients from ipsilateral frontal regions distant to the DLPFC, where no seizure-related activity could be measured. In 2 patients, no origin of pathological activity was found during the monitoring period. All patients were right-handed.

Recordings and selection of electrodes. Recordings were acquired using stainless steel subdural strip or grid electrodes (contact diameter: $4 \mathrm{~mm}$; center-to-center spacing: 10 $\mathrm{mm})$. All data were sampled at $1000 \mathrm{~Hz}$, referenced to linked mastoids, and bandpass filtered [0.01 Hz (6 dB/octave) to $300 \mathrm{~Hz}(12 \mathrm{~dB} /$ octave)] using the digital EPAS system (Schwarzer) and Harmonie EEG software (Stellate). The study was approved by the local medical ethics committee and was in accordance with the latest version of the Declaration of Helsinki; all patients signed written informed consent.

We selected one contact per patient in each ROI in which this patient was implanted (Fig. 1). This procedure was used to minimize interindividual variability, which would be higher if different numbers of electrodes would have been selected across patients. To guide the selection of contacts within DLPFC and DMPFC, we used MNI coordinates of a previous fMRI study of our group using the identical paradigm (Haupt et al., 2009) (contrast of conflict vs nonconflict trials in the phonetic task). For this purpose, all contacts were transferred into normalized MNI space using the Statistical Parametric Mapping software (SPM8, Wellcome Department of Imaging Neuroscience, London) and MRIcron (Chris Rorden's Neuropsychology Laboratory; www.mricro.com/ mricron), and the contact with the least distance to the reference point was calculated by taking the absolute value of the vector between the two coordinates. Afterwards, the location of the chosen contact within the ROI was confirmed by visual inspection of the non-normalized postimplantation MRI. In one patient with lateral frontal electrodes, no preimplantation MRI was available, which was however necessary for accurate normalization. We therefore chose the DLPFC electrode for this patient based on visual inspection and electrode labeling on the postimplantation MRI. DMPFC contacts were located at a distance between 3 and 12 $\mathrm{mm}$ (mean distance \pm SD: $8 \pm 4 \mathrm{~mm}$ ), DLPFC contacts between 9 and 36 $\mathrm{mm}(18 \pm 8 \mathrm{~mm})$ from the reference point.

To further assess the specificity of effects to the DMPFC and DLPFC, we analyzed conflict-related effects in a control region (i.e., the primary motor cortex contralateral to the responding hand). Handedness and 
A
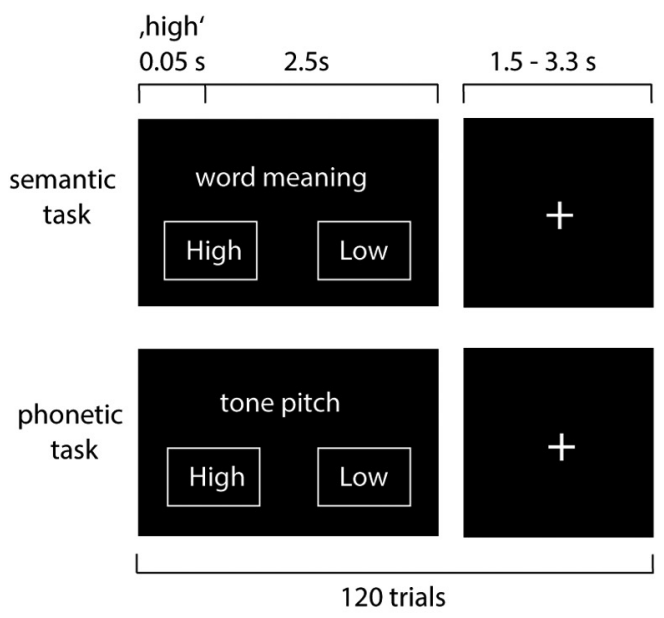

B

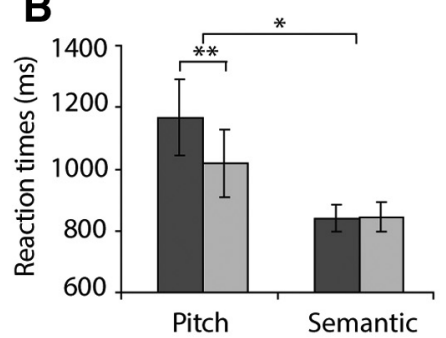

C

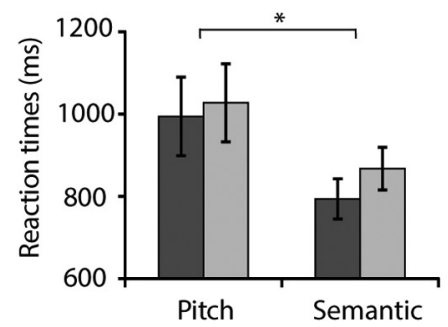

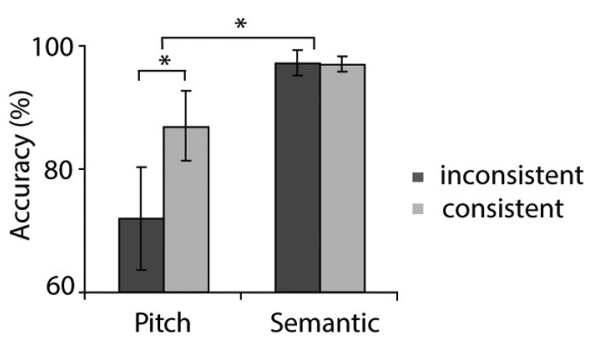

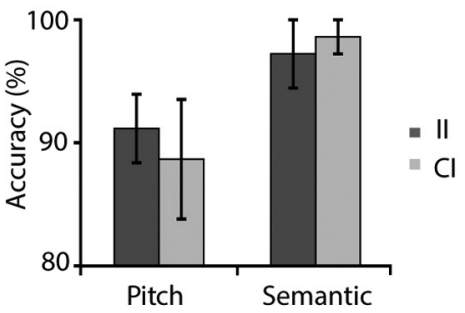

Figure 2. Paradigm and behavioral results. A, Experimental design. Patients responded to the words "high" and "low" spoken in a high or low pitch, resulting in consistent (nonconflict) and inconsistent (conflict) stimuli. Two tasks were performed: indication of word meaning (semantic task) or indication of tone pitch (phonetic task). Conflict was only expected to occur during the phonetic task. $\boldsymbol{B}$, Behavioral results related to conflict detection: Reaction times and accuracy for nonconflict and conflict trials. During the phonetic task, patients reacted significantly slower and less correctly to conflict words, whereas there was no behavioral effect of consistency during the semantic task. ${ }^{*} p<0.05$; ${ }^{* *} p<0.01$. C, Behavioral effects of consistency as a function of the previous trial (conflict adaptation). During both tasks, patients reacted faster and more accurately during two subsequent conflict stimuli (II) than to conflict trials preceded by nonconflict trials (Cl). ${ }^{*} p<0.05$.

electrode position allowed us to analyze this contrast in 2 patients. To this end, we converted all MNI coordinates into Talairach space and determined the nearest gray matter for each electrode (www.talairach.org). We chose all electrodes located within Brodmann area 4 and thereafter verified signal quality by investigating typical functional signatures of the primary motor cortex, that is, periresponse beta $(13-29 \mathrm{~Hz})$ power decreases and gamma $(30-100 \mathrm{~Hz})$ power increases followed by a postmovement rebound of beta power that exceeds baseline level (Crone et al., 1998; Pfurtscheller and Lopes Da Silva, 1999; Jurkiewicz et al., 2006; Miller et al., 2007). To this end, we performed a wavelet-transform of response-locked data (segmented - $2000 \mathrm{~ms}$ until $2000 \mathrm{~ms}$ around responses, independent of stimulus type) and extracted power values, which were expressed as a percentage of their frequency-specific baseline quantified as the averaged power across the full periresponse segment $(-2000$ to $2000 \mathrm{~ms})$. Anatomical selection resulted in three primary motor electrodes (one in the first, two in the second patient). Each of these three electrodes exhibited a pronounced beta power decrease before and around responses and a postresponse beta power rebound and was therefore included in the control analysis (see Fig. 6A, B). We did not find any increases in high-frequency power in the three available electrodes. This lack of high-frequency power increase is likely because highfrequency enhancements in the primary motor cortex are more focal than low-frequency power decreases (Crone et al., 1998; Miller et al., 2007). Thus, the limited number of available electrodes in the motor cortex might have restricted our ability to capture such focal highfrequency power effects.

Furthermore, we performed an additional control analysis minimizing remote effects to assure that our data analysis is not biased by covariance induced by the mastoid reference electrodes. It has previously been shown that averaged reference coherence estimates obtained from large electrode arrays posit the best approximation of theoretical reference-free coherence estimates (Nunez and Srinivasan, 2006). Therefore, we rereferenced each electrode to the global average of all frontal and interhemispheric nonpathological electrodes present in a patient (number of reference electrodes in each patient: 18-40). In particular, we tested the consistency of the main intraregional effects (DMPFC $\theta$ and DLPFC $\gamma$ power), as well as the interaction of these two effects (i.e., inter-regional CFC).

Experimental paradigm. We conducted an auditory Stroop paradigm similar to the one used in a previous fMRI study from our group (Fig. 2A)
(Haupt et al., 2009). Patients listened to the German equivalents of the English words "high" and "low," spoken in either high or low pitch. This results in trials where word meaning and phonetic information were consistent and those where the two stimulus characteristics were inconsistent. As an additional control, we added trials in which the German word for "good" was presented in either high or low pitch. The study comprised 240 trials in total and was split in two blocks of identical structure that differed in task instructions: In the semantic task, participants were asked to determine the word meaning ("low" or "high," regardless of pitch), whereas in the phonetic block, they were asked to identify whether the words were spoken in a low or high pitch (regardless of word meaning). Responses were indicated by counterbalanced left and right button presses with the dominant hand. Conflict should only occur for inconsistent stimuli in the phonetic task, when the more automatic response to the word meaning has to be suppressed to respond to the pitch. Control words could only be responded to during the phonetic task. In each block, patients listened to 40 inconsistent, 40 consistent, and 40 control words that were presented in randomized order. Participants were asked to respond as fast as possible. Most patients ( 9 of 12) conducted the semantic task first. Before the experiment started, patients performed a minimum of 10 practice trials to familiarize with the paradigm. Task instructions were displayed on the screen with every stimulus presentation.

Stimuli were presented for $0.5 \mathrm{~s}$, followed by a $2 \mathrm{~s}$ interval, during which instructions remained on the screen giving the patients additional time to respond. A jittered interstimulus interval (1.5-3.3 s) followed, in which a fixation cross was presented. Sound files were spoken by a male experimenter, digitized, and transposed to a high or low tone pitch (tone difference of a quint). The pitch was aligned using the Entropic Timescale Modification as included in the Goldwave audio editing software (http://www.goldwave.com/) to ensure an equal length of all sound files $(0.5 \mathrm{~s})$. Stimuli were presented using Presentation software (Version 0.71; Neurobehavioral Systems).

Analysis of behavioral data. Behavioral effects of conflict on reaction times and response accuracy were assessed using an ANOVA with the repeated measures "task" (phonetic vs semantic) and "consistency" (conflict vs nonconflict) using SPSS. Accuracy was defined as the ratio between the number of correct trials and total trials. Both incorrect and missing responses were counted as erroneous. To evaluate conflict adaptation (i.e., the beneficial effects of previous exposure to conflict on res- 
olution of a current conflict), we compared reaction times and accuracy between conflict trials preceded by correct conflict with those preceded by correct nonconflict trials. We therefore performed an ANOVA with the repeated measures "task" (phonetic vs semantic) and "previous trial" (conflict vs nonconflict). Three patients without two subsequent correct conflict trials during the phonetic task were excluded from the analysis of conflict adaptation effects. $p$ values were Huynh-Feldt corrected for inhomogeneities of covariance when necessary (Huynh and Feldt, 1976).

Time-frequency analyses. Artifacts were visually rejected using the Brain Vision Analyzer 2 software (Brain Products). Only trials without artifact were later used for further behavioral and EEG analysis. On average, 10 trials were discarded from each participant.

Stimulus-locked segmentation resulted in a final number of $30 \pm 12$ (mean \pm SD) correct conflict (range: 13-40) and $35 \pm 4$ (range: $31-39$ ) correct nonconflict trials for the DMPFC patients $(n=4)$. For the entire group of 12 patients, the number of correct trials was $28 \pm 11$ (range: $9-40$ ) conflict stimuli and the number of correct nonconflict stimuli $34 \pm 8$ (range: $12-40$ ). Further preprocessing and statistical analysis were performed using the FieldTrip toolbox (Oostenveld et al., 2011) (Donders Institute for Brain, Cognition, and Behavior, Nijmegen, Netherlands) for MATLAB (MathWorks) and our own MATLAB scripts. To minimize edge effects occurring after convolution with a wavelet kernel, we segmented the data into relatively large time intervals of $2 \mathrm{~s}$ before until $4.5 \mathrm{~s}$ after stimulus onset and discarded $1.5 \mathrm{~s}$ on each end of the segment after wavelet convolution. Segmented data were demeaned, line noise was removed, and the resulting data were convolved with a continuous complex Morlet wavelet with seven cycles. From the wavelettransformed signal, we extracted power and phase values between 4 and $100 \mathrm{~Hz}$ in $1 \mathrm{~Hz}$ steps.

Phase synchronization was calculated using the phase synchronization value (PSV) defined by Lachaux et al. (1999). Because unequal trial numbers may bias the results, we randomly selected as many trials from the condition with the higher number of trials as were measured in the condition with the lower number of trials. This was done 100 times, and results were averaged.

CFC between $\theta$ phases and $\gamma$ amplitude was analyzed by calculating phase synchronization between the low-frequency phase of the raw $\theta$ signal and the phase of fluctuations in high-frequency power. To calculate the latter, baseline-corrected high-frequency power values from 30 to $100 \mathrm{~Hz}$ were averaged across frequencies, and phases were extracted by calculating the angle of the Hilbert transformation of the time series of power values. After the adjustment of the number of trials per condition, we calculated PSVs between the time-series of low-frequency phases from 4 to $29 \mathrm{~Hz}$ and phases of fluctuations in high-frequency power with the method described above.

Power, PSV, and CFC were normalized with a baseline period of -0.5 to $-0.2 \mathrm{~s}$. To this end, all values were divided by mean values within the baseline period and are expressed as percentage of their frequencyspecific baseline. Using such a frequency-specific baseline ensures that effects in higher frequency ranges can be detected, which may otherwise be difficult because of the logarithmic decay of power with frequency. For further illustration of significant effects, we depicted $\gamma$ power values in significant time windows as a function of $\theta$ phases. To this end, we divided one cycle of an oscillation into 40 phase bins and allocated $\gamma$ power values within the significant time period into bins according to the present $\theta$ phase.

To assess a possible bias of inter-regional CFC by the enhancement of DMPFC $\theta$ power in an overlapping time-frequency window, we calculated correlations between these two measures. To this end, we mixed trials from both conditions within each patient and randomly selected as many trials from this data pool as were measured in the condition with the lower number of trials. This was performed 100 times; and for each subset of trials, we calculated DMPFC power and CFC values within the overlapping significant time window of both measures $(725-768 \mathrm{~ms})$. Thereafter, we computed Spearman correlation coefficients for each patient.

We defined the directionality of interactions between DMPFC and DLPFC by calculation of time- and frequency-resolved Granger causality (GC) using fieldtrip and the BSMART toolbox (Cui et al., 2008). To account for potential nonstationarity and to comprehend variations of GC in time, we applied GC to short data segments, which have been suggested to approximate stationarity (Ding et al., 2000; Barrett et al., 2012). To this end, we down-sampled the data to $250 \mathrm{~Hz}$ and used a model order of 12 to compute time-dependent sets of multivariate autoregressive coefficients in overlapping windows of $500 \mathrm{~ms}$ length (moving forward in $4 \mathrm{~ms}$ steps). The selected model order of 12 corresponds to a time lag of $48 \mathrm{~ms}$, similar to previous studies (Brovelli et al., 2004; Bollimunta et al., 2008), as a compromise between sufficient spectral resolution and the need to avoid overparameterization. Importantly, our results were robust against changes of model order in the range between 3 and 20 . Thereafter, GC $(4-100 \mathrm{~Hz})$ was calculated based on the spectral transfer matrices computed from the autoregressive coefficients. To assess the directionality of interactions between the DMPFC and DLPFC statistically, we compared the magnitude of Granger coefficients for each time point from 4 to $8 \mathrm{~Hz}$ for both directions (DMPFC $\rightarrow$ DLPFC; $\mathrm{DLPFC} \rightarrow$ DMPFC) during conflict trials using a cluster-based permutation test.

Statistical analysis. In general, we performed two main contrasts comparing neural activity during inconsistent with consistent stimuli during the phonetic and the semantic tasks. Whenever significant differences between inconsistent and consistent stimuli in the phonetic task were found, we furthermore compared neural activity during the latter two conditions to control stimuli. Because of the limited size of our sample, we refrained from factoring the laterality of recordings into our analysis. Particularly for DMPFC effects and the analysis of DMPFC-DLPFC interactions (4 patients; 3 left, 1 right hemisphere electrode), this would preclude any statistical evaluation of consistent effects across patients.

Statistics were performed using a nonparametric cluster-based approach (Maris and Oostenveld, 2007) comparing sum cluster $t$ values with surrogates obtained by randomly assigning condition labels to the average data from each participant. We used this procedure instead of shuffling between trials in each participant for three reasons: First, this constitutes the more appropriate procedure to test the null hypotheses that the differences between conditions are larger than interindividual differences. Second, we wanted to maintain a rigorous two-level statistical approach (random-effects analysis) throughout all analyses of our study because only this procedure treats "subject" as random factor and thus conceptually allows for generalization of our results. The analysis of each brain region yielded a different number of possible permutations because of the unequal number of patients with electrodes in each brain region. We computed 1000 permutations to test for effects in the DLPFC (12 patients) and the maximum number of possible 15 permutations for the 4 patients with DMPFC electrodes. In the latter case $(n=4)$, we only accepted the observed cluster-statistic as significant if it was greater than any of the possible surrogate test statistics. However, even in this case, our statistical procedure only allowed the conclusion that our observed cluster was located above the 93rd percentile (corresponding to $p<0.067$ ). For this reason, we set a significance threshold of $p<0.067$ whenever our analyses included four patients, and we report the $p$ value of observed clusters located above the $93 \mathrm{rd}$ percentile as $p=0.067$. In addition, we illustrate the location of the observed test statistic within the distribution of surrogate data for each analysis with four patients.

We tested the validity of this statistical procedure by allocating the position of the empirical test statistic within an alternative permutation distribution. The latter consisted of sum $t$ values obtained by randomly reassigning condition labels to single trials within each patient and thereafter calculating the respective measure (power, PSV, CFC). As a result, we obtained one surrogate average time-frequency dataset for each condition and patient. From each surrogate time-frequency dataset, we extracted sum $t$ values of the largest significant cluster as described above. Trial-level shuffling yielded a large number of possible permutations, of which we performed a subset of 1000 . Thereafter, the position of the empirical cluster in this permutation distribution was located, and $p$ values were assigned as described above (number of larger surrogate clusters divided by total number of permutations). This method comprised the advantage that the position of the empirical cluster above the 93rd percentile could be more precisely located using the high number of permutations. Thus, $p$ values $<0.067$ could be assigned. For GC con- 
trasts, this analysis could not be performed, as the original significant clusters were based on contrasting GC values during conflict stimuli between one direction and the other (DMPFC to DLPFC vs DLPFC to DMPFC). This would require shuffling of GC values on a single-trial level. However, this was not possible with the algorithm we applied, as it was based on a single multivariate autoregressive model for the entire dataset precluding estimations of singletrial values (Ding et al., 2000; Seth, 2010).

However, the cluster-based permutation procedure is of limited use for assessing effects in the high $(\gamma)$ frequency range, where fast fluctuations in power impede the formation of contiguous clusters across time. Therefore, we applied this cluster-based approach only to data between 4 and $29 \mathrm{~Hz}$. For the $\gamma$ frequency band, we conducted an ANOVA of power values averaged across longer time windows as a more sensitive measure to evaluate sustained effects of high-frequency activity (Fell et al., 2001; Axmacher et al., 2007). To assess the presence of separate sub-bands within the $\gamma$ frequency range $(30-100 \mathrm{~Hz})$, we averaged power values across all trials regardless of experimental conditions (i.e., phonetic and semantic task; conflict, nonconflict and control stimuli) in the DMPFC and DLPFC. In both regions, this analysis revealed two gamma power peaks: one centered on average at $\sim 46 \pm 11 \mathrm{~Hz}$ (mean \pm SD of frequency of maximal power) and the other centered at $\sim 77 \pm 14 \mathrm{~Hz}$, with slightly different peak frequencies for each patient (Fig. 3). Thus, we incorporated these sub-bands as a factor in our ANOVA and averaged power across nonoverlapping windows of $0.5 \mathrm{~s}$ duration within a low $(30-60 \mathrm{~Hz})$ and high $\gamma$ range $(61-100 \mathrm{~Hz})$, resulting in 12 values per patient and condition on which an ANOVA with the repeated measures "frequency band" (low vs high $\gamma$ ), "conflict" (conflict vs nonconflict), and "time window" was performed.

Specificity of effects. We selected one electrode per patient based on purely anatomical criteria for the following reasons: (1) this ensures an equal amount of data from each patient; (2) it avoids circularity, which may appear if contacts are selected based on functional criteria that are not completely orthogonal to the main analysis of interest; and (3) this allows us to conduct a strict random-effects (second level) analysis on averaged data from each patient.

However, from this analysis, we cannot gain information about the spatial specificity of the reported effects and the extent of cortex involved in conflict processing. To this end, we first tested the spatial specificity of the reported conflict-related power enhancements on the group level by contrasting conflict effects for electrodes that were near the target coordinate (local) compared with remote electrodes. Electrodes were defined as local when they were positioned within a particular radius around the target coordinate. This radius was defined as the range including a minimum of one electrode per patient (DLPFC: $36 \mathrm{~mm}$; DMPFC: $15 \mathrm{~mm}$ ). Thereafter, we divided all electrodes into two bins, depending on whether they were located inside or outside the chosen radius (DLPFC: $36 \mathrm{~mm}$; DMPFC: $15 \mathrm{~mm}$ ). In the DLPFC, 67 prefrontal electrodes (mean \pm SD distance from target coordinate: $34 \pm 16 \mathrm{~mm}$; distance range: $9-75 \mathrm{~mm}$ ), in the DMPFC, a total number of 16 interhemispheric electrodes (mean \pm SD distance from target coordinate: $20 \pm 11 \mathrm{~mm}$; distance range: $3-43 \mathrm{~mm}$ ) were available for this analysis. Thereafter, we averaged DLPFC $\gamma(30-100 \mathrm{~Hz}, 0-3000 \mathrm{~ms})$ and DMPFC $\theta$ power $(5-6 \mathrm{~Hz}$, $290-1250 \mathrm{~ms}$ ) of each electrode across frequencies and time points in conflict and nonconflict trials and performed a two-way ANOVA with the within-subject factor "conflict" and the between-subject factor "electrode distance."

Second, we were interested in the individual extent of cortex involved in conflict processing. To this end, we located conflict-related peakchanges for each patient and explored the spread of power enhancements to surrounding electrodes. In other words, power changes were measured numerically by power differences between conflict and nonconflict trials. Spatial limits were defined by identifying in each patient the elec- trode with the maximal distance from the peak electrode, up to which conflict-related power changes were still in the same direction.

Behavioral correlates of oscillatory changes. We further explored the functional role of conflict-related changes in power, phase synchronization, and CFC by correlating the magnitude of each significant finding with behavioral outcomes. First, we calculated intraindividual correlations between averaged preresponse values of each oscillatory measure and the respective reaction times across trials, supposing that a positive correlation would rather speak in favor of a functional role in conflict detection (i.e., convey information about the magnitude of conflict), a negative correlation for a role in conflict resolution. To this end, we first excluded outliers that exceeded the mean of the respective distribution by $>2$ SDs and thereafter calculated Spearman's correlation coefficients. To test significance on a group level, we tested Fisher-z-transformed correlation coefficients against the null hypothesis that the data derived from a normal distribution with zero mean (two-tailed $t$ tests). To identify the role of each measure in conflict adaptation, postresponse values in conflict trials were averaged (i.e., from the actual response up to $3 \mathrm{~s}$ ) and correlated with reaction times in the subsequent correct conflict trial. One patient with electrodes in the DMPFC and DLPFC and 2 patients with only DLPFC electrodes did not resolve two subsequent conflict trials correctly, leading to a reduced number of patients for the latter analysis.

As we calculated phase synchronization and CFC across trials, these measures cannot be used to calculate trial-by-trial correlations with reaction times. To obtain a trial-wise measure of synchronization $\left(\right.$ PSVtrial $\left._{j, \mathrm{k}}\right)$, we calculated phase differences between DMPFC and $\operatorname{DLPFC}\left(\Delta \theta_{j, \mathrm{k}}\right)$ in each trial $j$ for each time point $k$ and subtracted the average phase difference across trials for this specific point in time $\left(\mu_{\mathrm{k}}\right)$. To normalize negative and positive deviations from the mean phase difference, a cosine function was applied, resulting in values of 1 for a minimal phase difference of 0 and -1 for the maximal deviation of $\pi$ : PSVtrial $_{j, \mathrm{k}}=\cos \left(\Delta \theta_{\mathrm{j}, \mathrm{k}}-\mu_{\mathrm{k}}\right)$.

Thereafter, we investigated relationships between preresponse conflict-related oscillatory changes and subsequent accuracy. Whenever a trial-wise calculation of a measure was possible (i.e., power values), we averaged values in the significant frequency range before the actual response in each trial. Thereby, we included patients, who committed at least two errors in conflict trials (DMPFC: $n=3$; DLPFC: $n=10$ ). For phase synchronization and CFC, we analyzed differences between correct and incorrect conflict trials using the nonparametric cluster-based analysis described above (from 0 up to mean response time). There were not enough incorrect conflict trials after a correct conflict trial to enable us to establish relationships between postresponse oscillatory measures and the accuracy of conflict adaptation (i.e., accuracy in the next trial).

\section{Results}

\section{Behavioral data}

We first analyzed the effects of conflict processing on reaction times and accuracy (Fig. 2B). Slowing of reaction times due to 
conflict was specifically expected for the phonetic task because responding to the word meaning is more automatic than transforming the pitch into a semantic response. Therefore, the competing responses to inconsistent word meanings presumably induce conflict in the phonetic task. For reaction times, a repeated-measures ANOVA revealed significant effects of "task" (semantic vs phonetic; $\left.F_{(1,11)}=8.9, p<0.05\right)$ and "conflict" (consistent vs inconsistent; $F_{(1,11)}=7.2, p<0.05$ ), as well as a significant interaction $\left(F_{(1,11)}=10.1, p<0.01\right)$. Similarly, for response accuracy, we found effects of "task" $\left(F_{(1,11)}=7.5, p<\right.$ $0.05)$ and "conflict" $\left(F_{(1,11)}=5.3, p<0.05\right)$ and a trend toward an interaction between these two factors $\left(F_{(1,11)}=4.4, p=\right.$ 0.059). Post hoc $t$ tests revealed that conflict effects occurred exclusively during the phonetic task, where patients responded significantly slower $\left(t_{(11)}=3.3, p<0.01\right)$ and less correct $\left(t_{(11)}=\right.$ $-2.3, p<0.05)$ to conflict stimuli compared with nonconflict stimuli (semantic task: reaction times: $t_{(11)}=-0.3, p=0.8$; accuracy: $\left.t_{(11)}=0.1, p=0.92\right)$.

Furthermore, we investigated behavioral performance during the control condition compared with inconsistent and consistent stimuli during the phonetic task. In a previous study using an identical paradigm in healthy participants, we observed conflict (slower reaction times for inconsistent vs control stimuli), as well as facilitation (faster reaction times for consistent vs control stimuli) effects (Haupt et al., 2009). However, patient populations frequently respond slower and exhibit a higher interindividual variability in behavior than healthy subjects (Bruhn and Parsons, 1977; Mitchell et al., 1992; Axmacher et al., 2007). In this study, mean reaction times and error rates for control words were located between values for inconsistent and consistent stimuli. However, response times and accuracy were relatively variable between patients (mean reaction times \pm SD: inconsistent: $1164 \pm 431 \mathrm{~ms}$; consistent: $1017 \pm 381 \mathrm{~ms}$; control: $1138 \pm 449$ ms; mean accuracy \pm SD: inconsistent: $72 \pm 29 \%$; consistent: $87 \pm 20 \%$; control: $74 \pm 32 \%$ ). This high interindividual variance in behavioral measures in combination with a smaller group size might impede revealing the differences between control and inconsistent/consistent stimuli. Two paired-sample $t$ tests using a threshold of $p=0.025$ to correct for multiple comparisons showed that responses to consistent words were faster than for control stimuli (consistent vs control: $t_{(11)}=-3.6, p<0.01$ ), whereas inconsistent and control stimuli did not differ from each other (inconsistent vs control: $t_{(11)}=0.5, p=0.6$ ). Accuracy levels for control stimuli were not different from either conflict or nonconflict words (consistent vs control: $t_{(11)}=1.6, p=0.13$; inconsistent vs control: $t_{(11)}=-0.3, p=0.8$ ).

Next, we tested whether exposure to a conflict stimulus in one trial facilitates conflict processing in the subsequent trial (conflict adaptation; Fig. 2C). Thus, we analyzed reaction time and accuracy during conflict trials depending on whether the previous trial was a conflict trial. The factors "task" and "previous trial" exhibited significant main effects on reaction times in correct conflict trials (task: $F_{(1,8)}=6.8, p<0.05$; previous trial: $F_{(1,8)}=$ 8.6, $p<0.05)$, indicating that conflict trials were more easily solved if the preceding trial was a conflict trial as well. We found no significant effects of the previous trial type on accuracy.

\section{Effects of conflict on the power of activity in DMPFC and DLPFC}

As described in Materials and Methods, statistics for lowfrequency oscillations $(4-29 \mathrm{~Hz})$ were performed using a nonparametric cluster-based approach. This procedure controls effectively for multiple comparisons and allows one to work on an assumption-free basis regarding the sampling distribution under the null hypothesis (Maris and Oostenveld, 2007). However, for the assessment of sustained effects within the high $(\gamma ; 30-100$ $\mathrm{Hz}$ ) frequency range, this procedure is less well suited because the fast fluctuations in power disrupt the formation of continuous clusters. Instead, we conducted an ANOVA of power values averaged across longer time windows, which controls for multiple comparisons. This provides a more sensitive measure to evaluate sustained effects of high-frequency activity (Fell et al., 2001; Axmacher et al., 2007) (for a detailed description, see Materials and Methods).

In the DMPFC $(n=4)$, the nonparametric cluster-based permutation test on frequencies from 4 to $29 \mathrm{~Hz}$ revealed that $\theta$ power at 5 and $6 \mathrm{~Hz}$ was increased between 290 and $1251 \mathrm{~ms}$ for conflict compared with nonconflict trials during the phonetic task (Fig. $4 A-D ; p=0.067$; this is the lowest technically possible $p$ value; see Materials and Methods). There were no significant clusters in other frequency bands than $\theta$. Furthermore, the effect of conflict on DMPFC $\theta$ power was specific for the phonetic task, where conflict was behaviorally relevant. Contrasting inconsistent and consistent stimuli during the semantic task did not reveal any changes in power (Fig. $5 \mathrm{~A}$; largest cluster in the lowfrequency range: $p=0.33$; main effect of conflict for high frequencies: $\left.F_{(1,3)}=0.7, p=0.5\right)$. Consistent with our hypothesis, this effect started relatively soon after stimulus presentation, before a response was given.

We tested the behavioral relevance of DMPFC $\theta$ power enhancement by calculating a correlation between $\theta$ power and response times across single trials (correctly solved conflict trials only). Notably, only $\theta$ power before the actual response in each trial was taken into account. If $\theta$ power reflects conflict detection, one would expect more pronounced $\theta$ effects in trials with a higher degree of conflict (i.e., when reaction times are particularly long). Indeed, we found that the magnitude of conflictrelated $\theta$ power in the DMPFC correlates positively with reaction times during conflict trials $\left(t_{(3)}=3.9, p<0.05\right)$, but not during nonconflict trials $\left(t_{(3)}=1.0, p=0.38\right)$. These results suggest that $\theta$ activity in the DMPFC serves as a conflict detection signal.

In the DLPFC, we observed a sustained conflict-related increase in high-frequency power during the phonetic task. An ANOVA on $\gamma$ power values with the repeated measures "conflict," "frequency band," and "time" yielded a significant main effect of "conflict" $\left(F_{(1,11)}=6.6, p<0.05\right)$ but no interactions, indicating that conflict trials were associated with a sustained relative increase in $\gamma$ power between 30 and $100 \mathrm{~Hz}$ compared with nonconflict trials (Fig. $4 E, F$ ). We did not observe any effects of conflict on low-frequency DLPFC power during the phonetic task (largest clusters: inconsistent vs consistent: $p=0.35$ ). Similar to the DMPFC, conflict was not associated with any changes in power during the semantic task in the DLPFC (Fig. $5 B$; largest cluster in the low-frequency range: $p=0.43$; main effect of conflict for high frequencies: $\left.F_{(1,11)}=0.3, p=0.6\right)$.

Furthermore, there were no differences in $\gamma$ power between conflict and nonconflict stimuli before stimulus onset $\left(t_{(11)}=\right.$ $0.8, p=0.4$; tested on a time window from $-200 \mathrm{~ms}$ to stimulus onset; does not contain baseline period).

In contrast to the findings in the DMPFC, preresponse $\gamma$ power did not significantly correlate with reaction times. On the other hand, postresponse $\gamma$ power (averaged in a window between the response in each trial and $3 \mathrm{~s}$ after stimulus onset in this trial) in a correctly solved conflict trial predicted shorter reaction times in an upcoming correct conflict trial $t_{(8)}=-2.6, p<$ 0.05 ), which was not the case for upcoming nonconflict trials 


\section{DMPFC power effects}

A Conflict - non-conflict trials

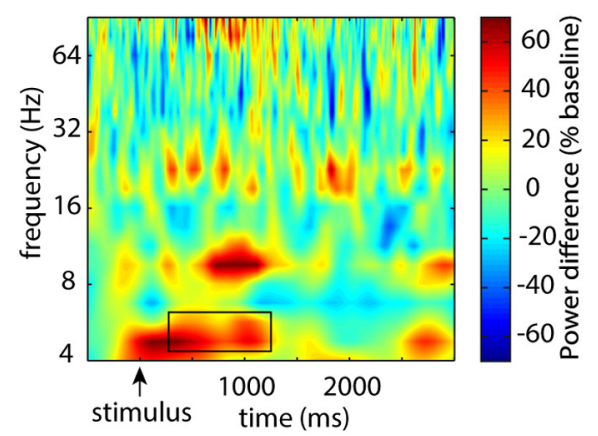

B

B Mean power at 5-6 Hz +/-SEM

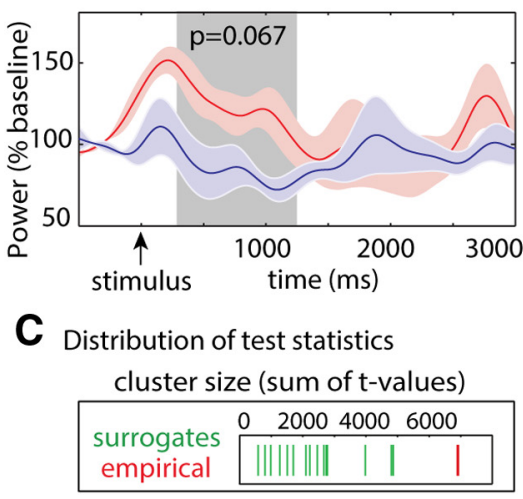

F Mean power at $30-100 \mathrm{~Hz}+/$ SEM

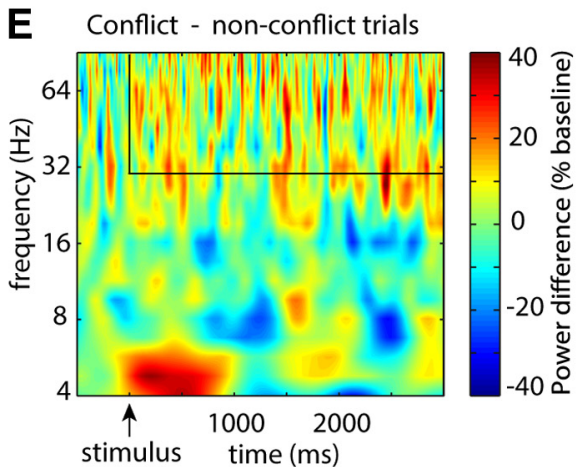

D Mean power at $5-6 \mathrm{~Hz}$ per patient

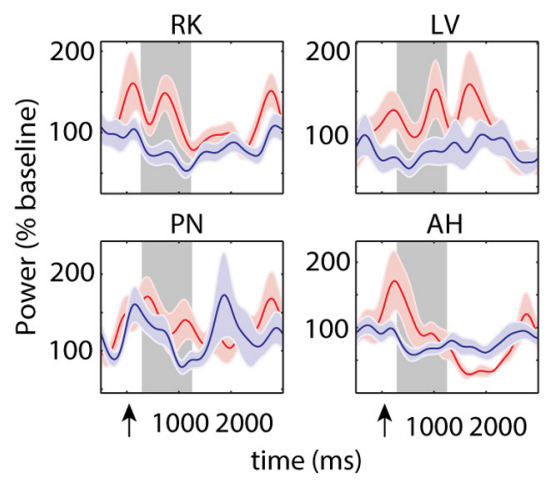

G Spatial specificity of effects

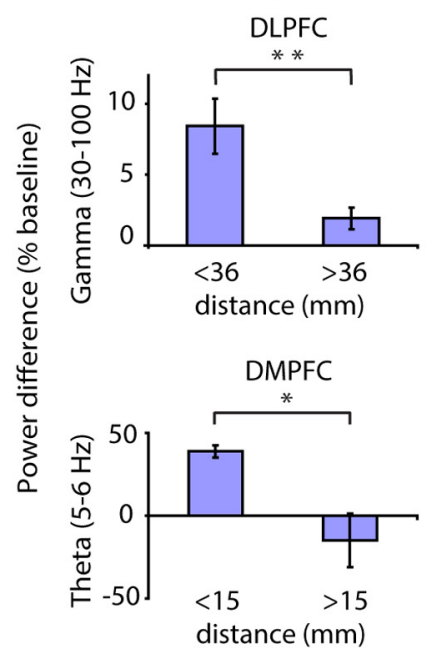

Figure 4. Conflict processing modulates preresponse DMPFC $\theta$ power and sustained DLPFC $\gamma$ power. $A$, $E$, Time-frequency plots during conflict processing (i.e., differences between conflict and nonconflict trials in the phonetic condition). Power differences are expressed in percentage of baseline power; significant clusters are highlighted. Conflict processing was associated with preresponse increases in DMPFC $\theta$ power and DLPFC $\gamma$ power enhancements throughout the trial. $\boldsymbol{B}, \boldsymbol{F}$, Time series of mean power \pm SEM across the respective significant frequencies for conflict (red) and nonconflict (blue) stimuli. For illustrative purposes, the DLPFC $\gamma$ power time series $(\boldsymbol{F})$ had been smoothed with a moving average of a $250 \mathrm{~ms}$ surrounding window. $\boldsymbol{C}$, Illustration of the location of the observed DMPFC $\theta$ power test statistic (red) within the distribution of surrogate values (green): sum of $t$ values. D, Plots illustrating conflict-related $\theta$ power in the DMPFC for all individual patients with electrodes in this region. For illustrative purposes, significant time periods are shaded in gray. G, Bar plot representing conflict-related power increases (conflict nonconflict items) in near compared with distant electrodes from the respective target coordinate across patients. ${ }^{*} p<0.05 ;{ }^{* *} p<0.01$.

$\left(t_{(10)}=-0.4, p=0.7\right)$. Postresponse $\gamma$ power in nonconflict trials predicted neither response times of upcoming conflict $\left(t_{(10)}=\right.$ $0.9, p=0.4)$ nor nonconflict stimuli $\left(t_{(10)}=0.6, p=0.6\right)$. Based on these findings, we conclude that preresponse DLPFC $\gamma$ power is increased during conflict trials and might play a role in conflict resolution, whereas postresponse $\gamma$ power mediates conflict adaptation.

Next, we compared DMPFC $\theta$ power and DLPFC $\gamma$ power changes during conflict and nonconflict stimuli to control stimuli (i.e., the word "good" presented in high or low pitch). In the DMPFC, cluster-based permutation tests showed that lowfrequency power $(4-29 \mathrm{~Hz})$ during control stimuli differed neither from conflict (largest cluster: $p=0.12$ ) nor from nonconflict stimuli (largest cluster: $p=0.13$ ). There were also no differences in high-frequency power during the control versus the conflict or nonconflict condition (main effect of conflict: conflict vs control: $F_{(1,3)}=0.15, p=0.7$; nonconflict vs control: $F_{(1,3)}=0.45, p=$ $0.5)$. Similarly, neither DLPFC low (conflict vs control: $p=0.56$; nonconflict vs control: $p=0.26$ ), nor high-frequency power during control stimuli was different from power during conflict stimuli (main effect of conflict: conflict vs control: $F_{(1,11)}=0.2$, $p=0.67$; nonconflict vs control $\left.F_{(1,11)}=3.2, p=0.1\right)$. These results are in accordance with the behavioral effects of control stimuli showing that mean latencies and accuracy of responses to control stimuli ranged between responses to inconsistent and consistent items but were relatively variable impeding the revelation of significant differences between stimuli types. We found a facilitation effect on reaction times, but contrasts between inconsistent and control stimuli did not reach significance. This high variance in behavioral measures is likely reflected in neural activity patterns underlying the processing of the different stimuli und thus may explain the nonsignificant differences between power values during control and inconsistent/consistent items. Similarly to behavioral outcomes, comparing activity during inconsistent and consistent items might therefore reveal the most pronounced effects. In the following contrasts, we thus focus on the comparison between 


\section{Semantic: inconsistent - consistent}

A

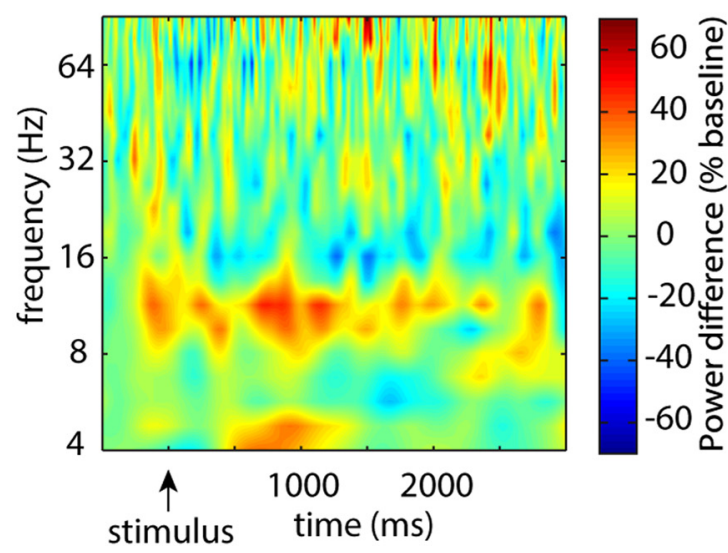

B

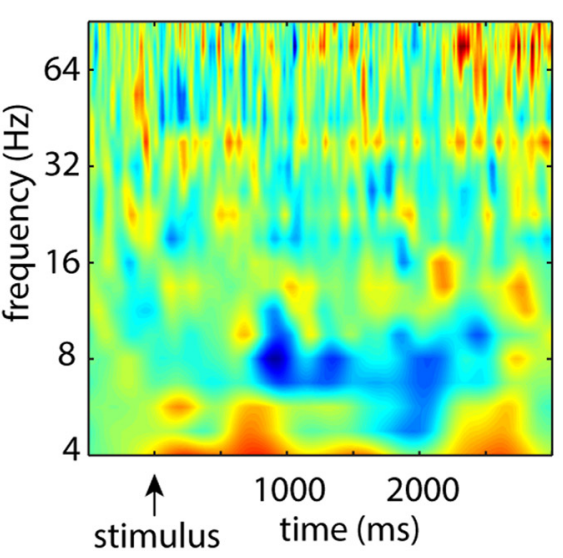

stimulus time (ms)
$-20$

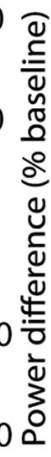

Figure 5. Inconsistent stimuli do not elicit DMPFC or DLPFC power changes during the semantic task. A, B, Time-frequency plots contrasting power values during inconsistent and consistent stimuli during the semantic task in the DMPFC and DLPFC. This contrast did not reveal any significant changes.

inconsistent (conflict) and consistent (nonconflict) stimuli, as described extensively in the literature (MacDonald et al., 2000; Kerns et al., 2004; van Veen and Carter, 2005).

\section{Spatial expanse of conflict-related power effects}

Comparison between local and remote conflict-related effects in the DMPFC and DLPFC revealed a different pattern of effect distribution within these two regions (Fig. 4G): For the DMPFC, a two-way ANOVA with the factors "conflict" and "distance" (electrodes within vs outside of a radius of $36 \mathrm{~mm}$ [DLPFC] or 15 $\mathrm{mm}$ [DMPFC]) showed no main effect of conflict, but a significant "conflict" $\times$ "distance" interaction $\left(F_{(1,14)}=4.7, p<0.05\right)$. Post hoc $t$ tests revealed a significant effect of conflict in the first distance bin with local electrodes $\left(t_{(4)}=10.9, p<0.001\right)$, but not for the second bin containing the more distant electrodes $\left(t_{(10)}=\right.$ $-0.9, p=0.38)$. In the DLPFC, we found a main effect of conflict $\left(F_{(1,65)}=18.7, p<0.001\right)$ and an interaction between the factors "conflict" and "distance" $\left(F_{(1,65)}=7.4, p<0.01\right)$. This suggests that conflict effects were present in both bins but were stronger in the first bin with local electrodes compared with the second bin with remote electrodes. Post hoc $t$ tests confirmed this result (first bin: $t_{(39)}=4.5, p<0.001$; second bin: $\left.t_{(26)}=2.4, p<0.05\right)$. These results indicate that $\theta$ power increases in the DMPFC were restricted to a relatively small radius of $15 \mathrm{~mm}$. In the DLPFC, effects were more widespread but significantly stronger in a radius of $36 \mathrm{~mm}$ around the target coordinate.

We also calculated the spread of conflict-related $\gamma$ power increases on an individual level. We found that power enhancements were restricted to $24 \pm 26 \mathrm{~mm}$ (mean distance from peak electrodes $\pm \mathrm{SD}$ ) in the DLPFC and $4 \pm 8 \mathrm{~mm}$ in the DMPFC.

Together, $\gamma$ power enhancements in the DLPFC were more widespread than $\theta$ effects within the DMPFC. This applied to group effects as well as the intraindividual spread of power increases. More extended effects on the group level might be related to a higher interindividual spatial variability in peak effects. However, individual peak electrodes were not clustered at a particular direction from the target coordinate. Instead, they were scattered in different directions around the target location. This indicates that the target position constitutes a good candidate for representing conflict-related effects for a second-level analysis.

\section{Control region: primary motor cortex}

In contrast to the DMPFC and DLPFC, we did not find any effects of conflict on power values in the motor cortex (Fig. 6C-F). The low number of patients with respective electrodes $(n=2)$ did not allow us to perform a cluster-based group analysis or repeatedmeasures ANOVA. However, we investigated conflict-related effects on power in each of the 2 patients separately by performing a cluster-based permutation test based on shuffling trials across conditions. This did not reveal any significant clusters, either in low frequencies $(4-29 \mathrm{~Hz}$; Patient 1: all clusters, $p>0.2$; Patient 2: all clusters, $p>0.28)$, or in high frequencies $(30-100 \mathrm{~Hz}$; Patient 1: all clusters, $p>0.27$; Patient 2: all clusters, $p>0.16$ ). Figure $6 E, F$ illustrates the distribution of $\theta$ and $\gamma$ power values across trials.

\section{Phase synchronization}

Four patients were implanted with electrodes in both the DMPFC and the DLPFC, which enabled us to investigate neural communication between these areas. As the different temporal patterns of power effects in DMPFC and DLPFC within the trial indicated that communication between DMPFC and DLPFC might change in relation to responses, we evaluated effects of conflict on phase synchronization and other measures of inter-regional interactions by means of nonparametric cluster-based permutation tests in two time windows: (1) from stimulus onset up to the mean reaction time to conflicts $(1164 \mathrm{~ms})$; and (2) from the mean reaction time to conflicts up to the end of the trial $(3000 \mathrm{~ms})$. Following the results of the power analysis, we focused on phase synchronization in the $\theta$ band at 5 and $6 \mathrm{~Hz}$. We also tested for effects of conflict in the alpha $(9-12 \mathrm{~Hz}$; largest cluster: $p=0.07)$ and beta (13-29 Hz; largest cluster: $p=0.2)$ frequency bands. There were no significant effects in any other frequency band than $\theta$. We found that conflicts were associated with increased phase synchronization at $5 \mathrm{~Hz}$ before the average response latency (Fig. $7 A-D ; 640-712 \mathrm{~ms}, p=0.067$ ) [cluster-based permutation statistics], as well as in several late clusters after the average response latency (1480-1761 ms, 2103-2365 ms, and 2521-2847 ms; all $p=0.067)$. Thereby, preresponse phase synchronization in the significant interval was related to the correct solution of the current trial: A nonparametric cluster analysis comparing prere- 


\section{Primary motor cortex: response- and stimulus locked activity}

A Patient 1: across conditions

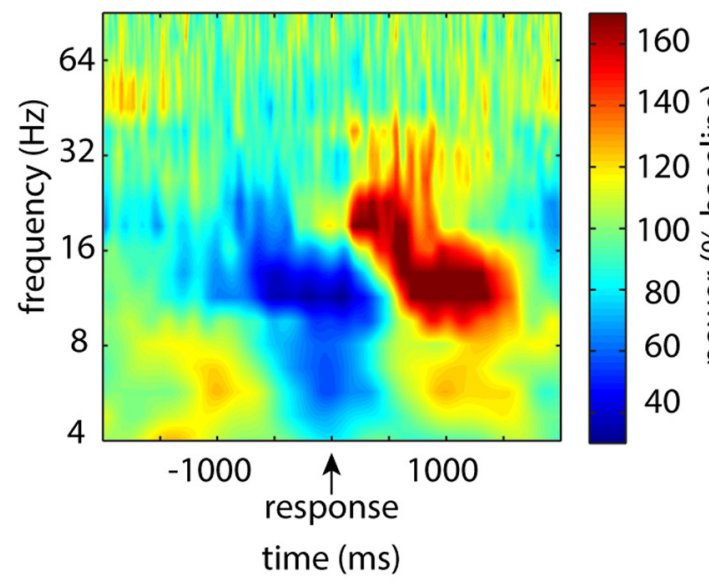

C Patient 1 : conflict - non-conflict

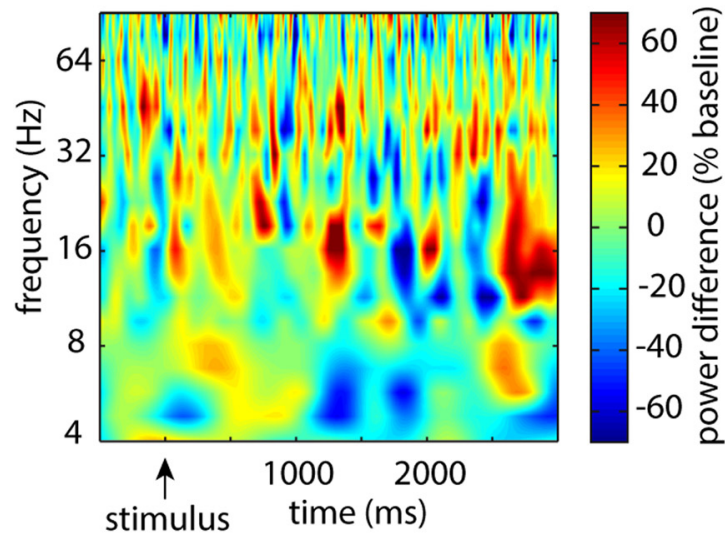

E Mean theta power $(5-6 \mathrm{~Hz}) \pm \mathrm{SEM}$

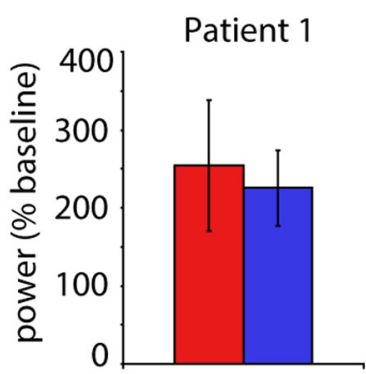

Patient 2

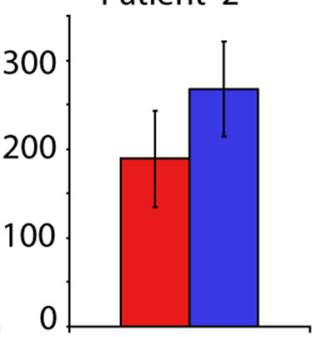

inconsistent
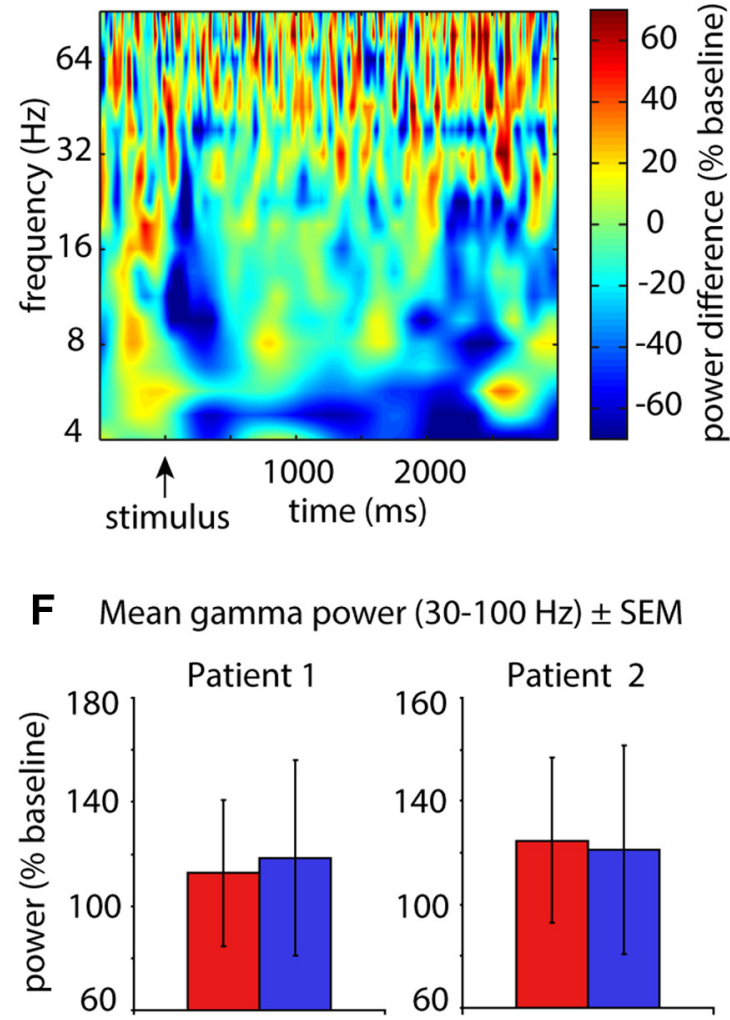

F Mean gamma power $(30-100 \mathrm{~Hz}) \pm \mathrm{SEM}$

consistent

Figure 6. Conflict was not associated with power changes in the motor cortex. $A, B$, Response-locked analysis of oscillatory activity in the primary motor cortex. Signal quality and electrode location were confirmed by response-locked analyses (independent of stimulus type). All motor electrodes exhibited typical functional signatures of the primary motor cortex (a pronounced periresponse beta power decrease and postresponse beta power increase). It should be noted that power values were calculated independent of experimental conditions and are shown as percentage of their baseline. As the images do not represent differences between conditions as all other contrasts (inconsistent - consistent), values fluctuate $\sim 100 \%$ (baseline level) instead of zero. $\boldsymbol{C}, \boldsymbol{D}$, Stimulus-locked analysis of oscillatory activity in the primary motor cortex. Time-frequency-resolved illustration of conflict-associated changes of power in the motor cortex of 2 patients. Within-subjects statistics did not reveal any significant clusters. $\boldsymbol{E}, \boldsymbol{F}$, Bar plot illustrating the distribution of $\theta$ and $\gamma$ power values in both conditions (inconsistent vs consistent) across trials.

sponse phase-synchronization at $5 \mathrm{~Hz}$ between correct and incorrect conflict trials revealed a significant positive cluster from 671 to $745 \mathrm{~ms}(p=0.067)$. These results indicate enhanced DMPFCDLPFC communication during conflict trials, which predicts correct conflict resolution.
Cross-frequency coupling (CFC)

Within the DLPFC, cluster-based permutation statistics revealed an increased modulation of $\gamma$ power by $\theta$ phase for conflict compared with nonconflict trials in an early time window (Fig. 7E-G; significant cluster for low-frequency phases of 4 and $5 \mathrm{~Hz}$ oscil- 


\section{Stimulus-locked DMPFC - DLPFC phase synchronization}

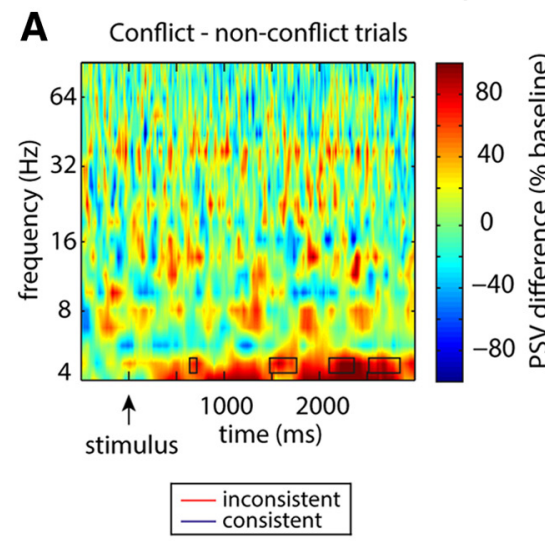

B Mean PSV at $5 \mathrm{~Hz}+1$-SEM

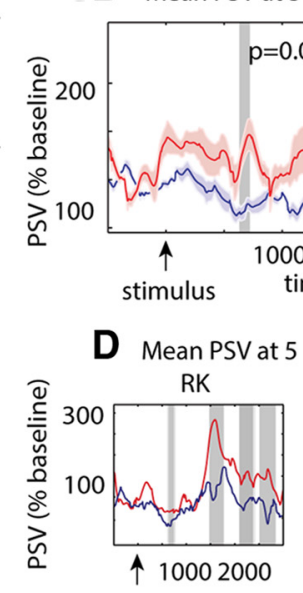

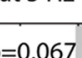
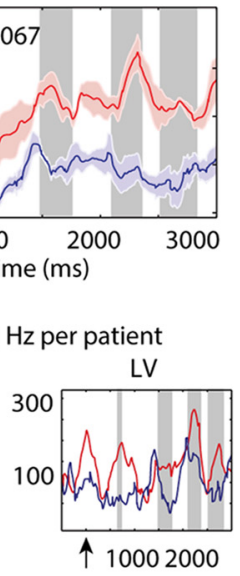

C Permutation distribution

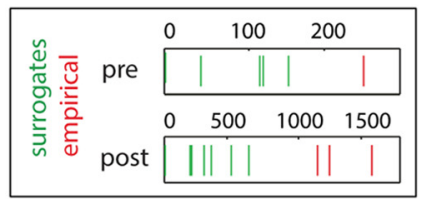

cluster size (sum of t-values)

\section{Stimulus-locked DLPFC cross-frequency coupling}

E Conflict-non-conflict trials

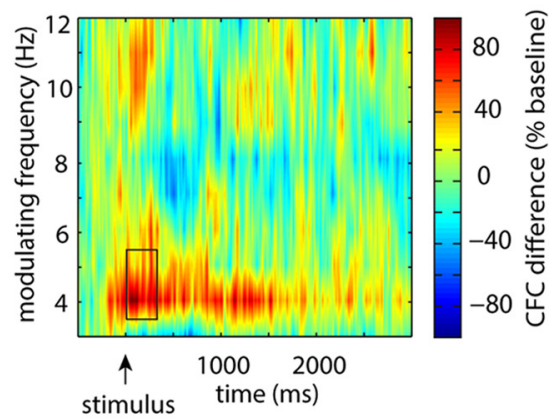

F Mean CFC at 4-5 Hz +/- SEM

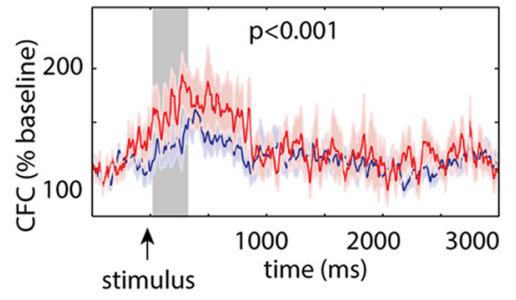

Stimulus-locked DMPFC - DLPFC cross-frequency coupling

H Conflict - non-conflict trials

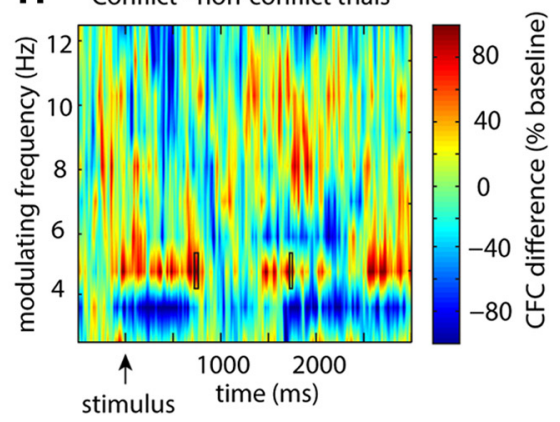

K

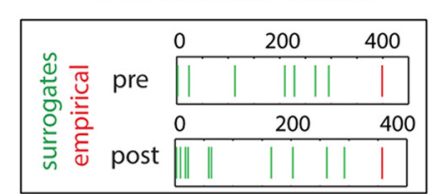

cluster size (sum of t-values)

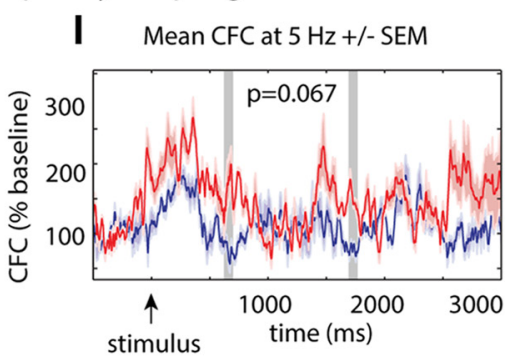

L Mean CFC at $5 \mathrm{~Hz}$ per patient
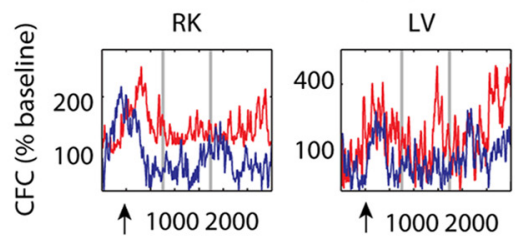

G Phase-dependent gamma power

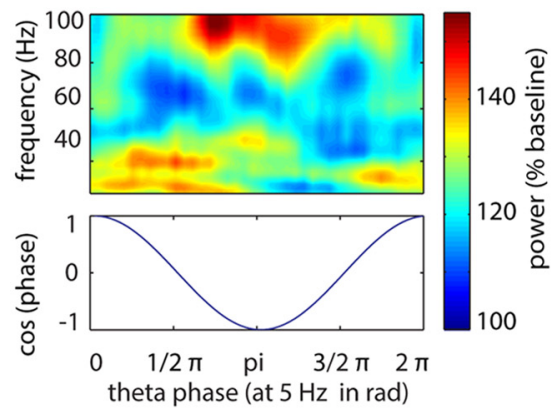

$J$ Phase-dependent gamma power
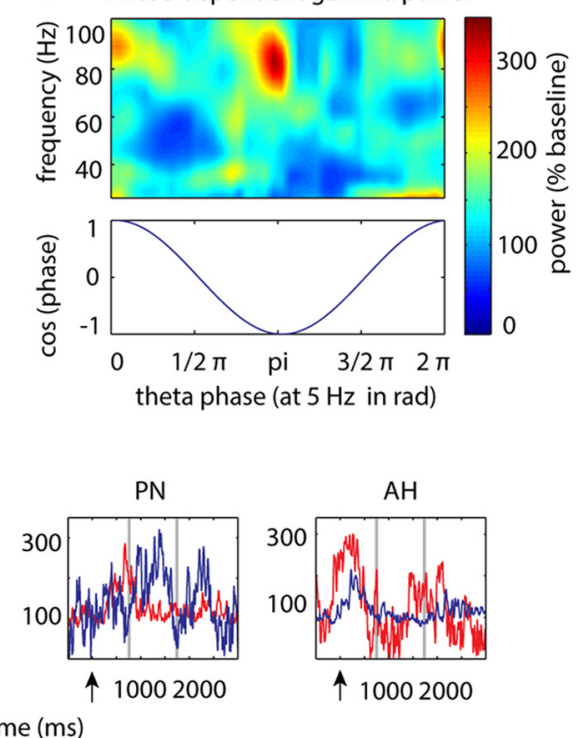

time (ms)

Figure 7. Conflict-related changes in CFC within the DLPFC and phase synchronization and CFC between DMPFC and DLPFC. Significant clusters are highlighted, and the location of the observed test statistic (red) within the surrogate distribution (green) is shown for all analyses with $n=4$ patients and $n=15$ possible surrogate permutations ( $\boldsymbol{C}, \boldsymbol{K}$; cluster size as sum of $t$ values). When $<15$ clusters are indicated, the remaining permutations did not reveal any significant clusters. $A, B, D$, Time-frequency plots of DMPFC-DLPFC PSVs during conflict processing (conflict vs nonconflict trials). Conflict was associated with synchronization increases at $5 \mathrm{~Hz}$ before and after average response latency (highlighted). $\boldsymbol{E}-\boldsymbol{G}$, Intraregional CFC within the DLPFC during conflict compared with nonconflict trials. During conflict, early DLPFC $\gamma$ power was transiently locked to DLPFC $\theta$ phases. $\boldsymbol{E}$, Time-dependent magnitude of the modulation of $\gamma$ power by low-frequency phases. $\boldsymbol{F}$, Time-dependent CFC at $5 \mathrm{~Hz}$, where significant conflict-associated changes had been found. G, DLPFC $\gamma$ power during conflict trials as a function of low-frequency phases within the significant time window. $\boldsymbol{H}-\mathbf{J}, \mathbf{L}$, Conflict-related increase in inter-regional CFC between DMPFC $\theta$ phase and DLPFC $\gamma$ power before and after average response latency. 
A Stimulus-locked Granger causality

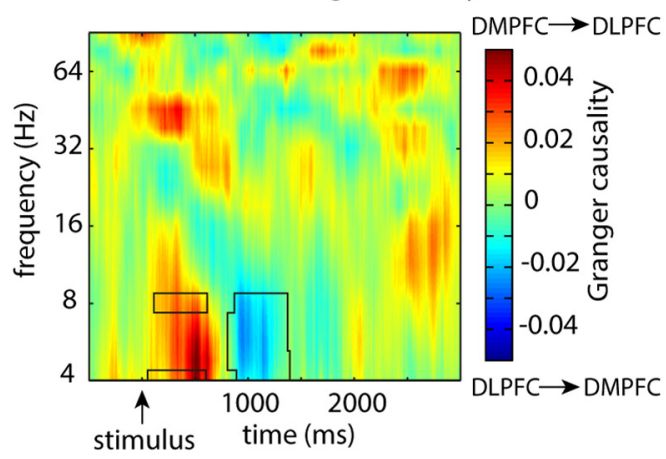

B Mean GC at $4-8 \mathrm{~Hz}+/$ - SEM

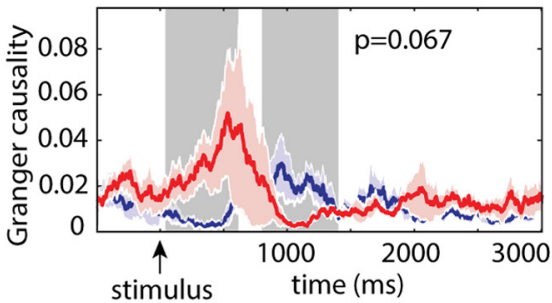

C Permutation distribution

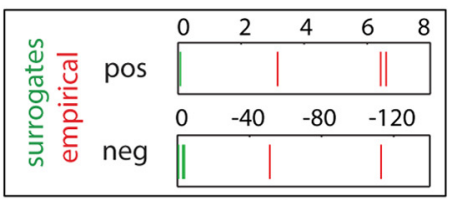

cluster size (sum of t-values)

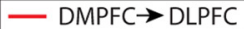
- DLPFC $\rightarrow$ DMPFC

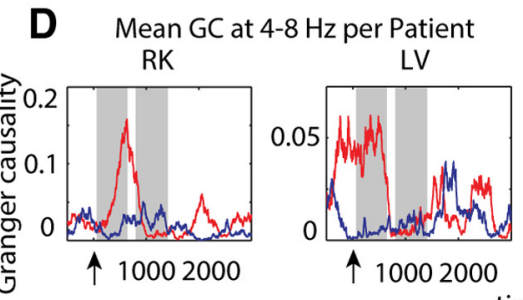

time (ms)
PN

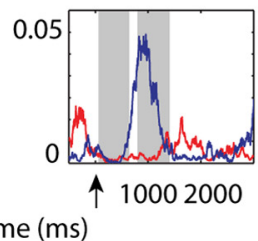

$\mathrm{AH}$

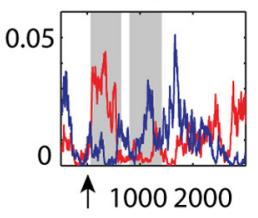

Figure 8. GC analyses. Directionality of interactions between DMPFC and DLPFC during conflict processing. $A$, Time- and frequency-resolved GC ([DMPFC $\rightarrow$ DLPFC $]-[D L P F C \rightarrow D M P F C])$. Whereas early in the trial, oscillatory activity in the $\theta$ range within the DMPFC predicted DLPFC activity significantly better than the other way around, the predominant direction of information flow reversed closer to the mean response time. $\boldsymbol{B}$, Time series of GC between the DMPFC and DLPFC (mean \pm SEM) between 4 and $8 \mathrm{~Hz}$. For illustrative purposes, significant time periods are shaded in gray. C, Position of empirical cluster sizes (red) within the distribution of 15 cluster sizes obtained from random permutations (green): as measured in sum of $t$ values. When $<15$ random test statistics are shown, the remaining permutations did not reveal any significant clusters. D, Plots showing conflict-related GC from 4 to $8 \mathrm{~Hz}$ for each patient separately.

lations between 10 and $331 \mathrm{~ms} ; p<0.001)$. Previous studies have shown that spontaneous fluctuations of peristimulus DLPFC activity influence subsequent accuracy or reaction times to conflict stimuli in Stroop paradigms (MacDonald et al., 2000; Stern and Mangels, 2006, Coste et al., 2011). Indeed, we found that the preresponse CFC within the DLPFC was predictive for the correct solution of conflict: A cluster analysis revealed significantly higher CFC in correct compared with incorrect conflict trials from 361 to $464 \mathrm{~ms}$ at $4-5 \mathrm{~Hz}(p<0.05)$. There was no correlation with reaction times. We did not find any intraregional CFC in the alpha (9-12; largest cluster: $p=0.31)$ or beta $(13-29 \mathrm{~Hz}$; largest cluster: $p=0.08$ ) frequency band.

For inter-regional CFC, we found an increased modulation of DLPFC $\gamma$ power by DMPFC $\theta$ phases at $5 \mathrm{~Hz}$ for conflict versus nonconflict trials before and after mean reaction time (Fig. $7 \mathrm{H}-\mathrm{L}$; $725-768 \mathrm{~ms}, p=0.067$ and $1719-1759 \mathrm{~ms}, p=0.067$ ). Thereby, preresponse inter-regional CFC was related to conflict resolution. A cluster analysis comparing $\mathrm{CFC}$ between correct and incorrect conflict trials at $5 \mathrm{~Hz}$ revealed a significant increase of CFC for correct versus incorrect trials between 603 and $630 \mathrm{~ms}$ $(p=0.067)$. Furthermore, postresponse CFC (between the actual response and $3 \mathrm{~s}$ ) between the DMPFC and DLPFC was relevant for the preparation for an upcoming conflict: Trial-by-trial correlations showed that increased postresponse CFC was associated with faster reaction times in an upcoming conflict trial $\left(t_{(2)}=\right.$ $-4.5, p<0.05)$. Additional analyses revealed that the increase in inter-regional CFC could not be explained by concomitant increases in DMPFC $\theta$ power (see Materials and Methods): We did not find a correlation between these two measures during the significant time periods $\left(725-768 \mathrm{~ms}\right.$ and $1719-1759 \mathrm{~ms} ; t_{(3)}=$ $\left.-0.2, p=0.8 ; t_{(3)}=0.04, p=0.96\right)$. We also analyzed effects of conflict in the alpha $(9-12 \mathrm{~Hz}$; largest cluster: $p=0.39)$ and beta (13-29 Hz; largest cluster: $p=0.45$ ) frequency range. However, we did not find any clusters in the latter frequency ranges. Thus, inter-regional CFC effects were specific to the $\theta$ band.

Statistically, the conflict-related increase of inter-regional CFC occurred only during very short time windows. This may be explained by two factors. First, we chose a purely data-driven approach for our analysis working with maximally authentic data. To this end, we restricted the use of filters to a necessary minimum (i.e., line-noise removal). Therefore, all measures calculated on the basis of high-frequency oscillations, such as highfrequency power and $\theta-\gamma$ CFC, are naturally subject to fast fluctuations. Second, we used a rigorous nonparametric clusterbased permutation test for the assessment of differences between conditions. This test corrects for factors, such as the influence of noise on the data, because effect sizes of the observed clusterstatistic are reported in regard to their position in the permutation distribution, which includes all possible random clusters.

These data provide evidence that $\theta$-phase to $\gamma$-power CFC within the DLPFC and between DMPFC and DLPFC is related to both conflict resolution of the current and conflict adaptation to the next trial.

\section{Granger causality (GC)}

Finally, the directionality of interactions between DMPFC and DLPFC was calculated by means of a time- and frequencyresolved GC analysis. Only correct conflict trials were considered for this analysis. Cluster-based permutation statistics revealed that the directionality of DMPFC-DLPFC interactions changed over time. Before the average response time, DMPFC activity predicted DLPFC activity significantly better than the other way around in three time clusters (Fig. 8; at 4 Hz: 310-314 ms and 350-354 ms; at $8 \mathrm{~Hz}$ : at $366 \mathrm{~ms}$; all $p=0.067)$, indicating more pronounced causal influence of the DMPFC on the DLPFC than vice versa. This directionality was reversed in two later clusters $(4-8 \mathrm{~Hz}$ : 1126-1158 ms; 5-8 Hz: 1058-1074 ms; both $p=0.067)$. Because GC was computed based on autoregressive coefficients in sliding time windows of $500 \mathrm{~ms}$, each point in the significant clusters marks the center point of a $500 \mathrm{~ms}$ window in which the activity in one region predicts activity in the other region. Therefore, information transfer in the $\theta$ range from the DMPFC to the DLPFC was higher from 60 to $616 \mathrm{~ms}$, and in the reverse direction (DLPFC $\rightarrow$ DMPFC) from 808 to $1408 \mathrm{~ms}$. This pattern of directionality was specific for correct conflict trials: In incorrect conflict trials, both directions were equally present before mean 
response time. However, around and after mean response time, incorrect conflict trials were associated with six positive clusters indicating increased information flow from DMPFC to DLPFC (896-1720 ms, all $p=0.067)$. There were no effects of conflict on GC in the alpha $(9-12 \mathrm{~Hz}$; largest cluster: $p=0.12)$ and beta (13-29 Hz; largest cluster: $p=0.75$ ) frequency band. For correct and incorrect nonconflict trials, we did not find any asymmetries in directionality after the response.

\section{Analysis of rereferenced data}

The analysis of intraregional (power) effects, as well as interregional interactions (inter-regional CFC) with rereferenced data showed that conflict-associated effects were relatively robust against changes of the reference electrode. For the averagereferenced data, we found $\theta$ power increases in a very similar frequency and time window in the DMPFC as for the mastoidreferenced data $(5 \mathrm{~Hz}, 94-604 \mathrm{~ms}, p=0.067 ; 815-857 \mathrm{~ms}, p=$ $0.067 ; 1137-1236 \mathrm{~ms}, p=0.067)$. In the DLPFC, conflict-related $\gamma$ power increases were reduced $(30-100 \mathrm{~Hz}$, main effect conflict: $\left.F_{(1,11)}=2.2, p=0.16\right)$ when electrodes were rereferenced to the global average. However, this is probably the result of the relatively widespread increase in $\gamma$ power across the DLPFC during conflict (see above). As the data were rereferenced against all frontal and interhemispheric electrodes, the presence of conflict effects in a large portion of reference electrodes would diminish the measured effect in the analyzed electrode. Furthermore, inter-regional interactions between DMPFC $\theta$ oscillations and DLPFC $\gamma$-band activity (inter-regional CFC) were reproducible with rereferenced data $(746-752 \mathrm{~ms}, p=0.067 ; 1683-1730 \mathrm{~ms}$, $p=0.067)$. This indicates that our results are most likely not explainable by covariance induced by the mastoid electrodes.

\section{Response locked analysis}

Here, we report stimulus-locked analyses of our data. The relation of each subprocess of conflict processing to responses plays an important role in understanding the behavioral role of the underlying neural correlates. However, response-locked analyses are particularly difficult in populations with highly variable reaction times. In contrast to healthy subjects, response times of our patients were highly variable. This was visible across patients (reaction times: pitch inconsistent: $1164 \pm 431$ ms [mean \pm SD]; pitch consistent: $1017 \pm 380 \mathrm{~ms}$ ), as well as within individual patients: mean $\pm \mathrm{SD}$ of response times of individual patients during conflict trials ranged from $564 \pm 106$ to $2281 \pm 916 \mathrm{~ms}$. In our study, we found different roles of preresponse and postresponse neural activity for conflict processing. However, despite these findings, conflict-related processes are probably not timeor phase-locked to responses if additional interfering processes contribute to variable delays in response time. The latter would diminish effect size in a response-locked analysis. Bases on our previous iEEG analyses, we therefore chose a stimulus-locked analysis. However, we additionally performed a response-locked analysis, which did not reveal any significant differences between conflict and nonconflict stimuli.

\section{Mean-shuffled versus trial-shuffled surrogate analysis}

We tested the robustness of our results by locating the position of each significant cluster within a second distribution of surrogate statistics originating from random within-subject trial-label permutations. Within the distribution of 1000 surrogate clusters based on trial-level condition shuffling, all except one original cluster reached the significance threshold of $p<0.05$ : DMPFC $\theta$ power: $p=0.045$; phase synchronization: $p=0.09$ (preresponse cluster), $p=0.02$ to $p=0.03$ (3 postresponse cluster); interregional CFC: $p=0.01$ (preresponse cluster), $p=0.04$ (postresponse cluster); intraregional CFC: $p=0.01$. Our results indicate that, despite the small number of possible permutations, the random permutation of within-subject means provides a valid procedure to reject the null hypothesis in this experiment.

\section{Discussion}

Using intracranial EEG recordings during an auditory Stroop task, the present study reveals the fine-grained neural communication pattern between medial and lateral prefrontal cortex during conflict detection, resolution, and adaptation. It should be noted that the position of lateral frontal electrodes differed between left and right hemispheres, with left being more ventral (IFG) and right being more dorsal (M/SFG), as electrode selection was guided by the anatomical location of activation patterns observed in a previous fMRI study using an identical paradigm (Haupt et al., 2009). As extensively reported in the literature (MacDonald et al., 2000; Kerns et al., 2004; van Veen and Carter, 2005), we compared neural activity during stimuli containing two inconsistent to stimuli containing two consistent stimulus features (i.e., conflict vs nonconflict stimuli). Originally, we obtained additional recordings for control stimuli. However, typical for studies in patients, we observed a high variability in response accuracy and latencies (Bruhn and Parsons, 1977; Mitchell et al., 1992; Axmacher et al., 2007), impeding the revelation of behavioral differences between control stimuli and conflict/nonconflict items. Accordingly, we focused our analysis on the contrast with the most pronounced behavioral effects (i.e., conflict vs nonconflict stimuli).

\section{Functional role of DMPFC $\boldsymbol{\theta}$ oscillations and DLPFC $\boldsymbol{\gamma}$ - band activity}

Our results suggest that conflict detection starts $\sim 290 \mathrm{~ms}$ after stimulus presentation and is reflected by an increase in $\theta$ power in the DMPFC (Fig. $4 A-D$ ). This $\theta$ power increase was more related to conflict detection than to conflict resolution, as supported by its early appearance and by its positive correlation with reaction times: Trials that elicited a strong conflict (as indicated by longer reaction times) showed enhanced DMPFC $\theta$ power. If $\theta$ power in the DMPFC supported conflict resolution, a negative relation would have been predicted. This finding corroborates the results of prior scalp EEG studies reporting medial prefrontal $\theta$ power increases during conflict items (Hanslmayr et al., 2008; Staudigl et al., 2010; Pastötter et al., 2012) associated with slower response times (Cavanagh al., 2012).

In contrast to DMPFC, the DLPFC might rather play a role in conflict resolution and adaptation. We found a sustained broadband $\gamma$ power increase $(30-100 \mathrm{~Hz})$ in response to conflicting stimuli that was evident before as well as after the mean response time (Michels et al., 2010; Koga et al., 2011; Swann et al., 2013) (Fig. $4 E, F$ ). Interestingly, we did not only observe an increase in $\gamma$ power during processing of inconsistent items compared with baseline but also a decrease during processing of consistent stimuli. Thus, preparation for an upcoming stimulus appears to require more extensive resources than processing of a consistent stimulus.

Thereby, $\gamma$ power enhancements before and after mean response time seemed to serve different behavioral functions. We did not observe any behavioral correlations for preresponse $\gamma$ power enhancements. However, early intraregional CFC (Canolty et al., 2006; Axmacher et al., 2010) increased during conflict trials (Fig. 7E-G) and was significantly higher for correct 
versus incorrect trials. Thus, an early enhanced rhythmic modulation of preresponse broadband $\gamma$ power by $\theta$ oscillations seems to be beneficial for the correct solution of conflicts. The early appearance $(10-331 \mathrm{~ms})$ of this effect indicates that it reflects preparatory processes required for successful completion of the task.

In contrast, postresponse $\gamma$ power increases in conflict trials predicted shorter response times in the following conflict trials, suggesting that the DLPFC also prepares the system for the next trial after the response was given, thus mediating conflict adaptation (Mansouri et al., 2007, 2009). This correlation between postresponse $\gamma$ power and subsequent reaction time was specific to two subsequent conflict trials. This speaks in favor of a conflict-specific adaptive process reflected by $\gamma$ power increases, rather than conflict-unspecific repetition priming as suggested previously (Mayr et al., 2003).

Importantly, our control analyses showed that the latter conflict-related effects were specific to the DMPFC and DLPFC. First, we observe that conflict-related power enhancements decrease with increasing distance from the target coordinate (Fig. $4 G)$. Thereby, effects in the DLPFC were more widespread than in the DMPFC. Second, other task-relevant regions of the frontal cortex, such as the primary motor cortex, did not exhibit any conflict-associated changes in power (Fig. $6 A-F$ ).

\section{Inter-regional communication during conflict processing}

A central question is how the two prefrontal brain regions communicate with each other to eventually resolve conflict. Here, we show that DMPFC-DLPFC $\theta$ phase synchronization occurs after the onset of the DMPFC conflict detection signal, $\sim 600 \mathrm{~ms}$ after stimulus presentation (Fig. $7 A-D$ ). Importantly, this preresponse phase synchronization effect was more pronounced for correct than for incorrect trials. Furthermore, the results of the GC analysis (Fig. 8) show that the medial prefrontal cortex transmits a conflict signal to the lateral prefrontal cortex in the $\theta$ frequency range. These results speak in favor of the conflict monitoring model by Botvinick et al. (2001) rather than models assuming an opposite direction of information flow (Morishima et al., 2010).

But how are these conflict-related signals in the $\theta$ frequency range related to the $\gamma$ power effect in the lateral PFC which predict successful conflict resolution and adaptation? Inter-regional CFC analysis showed that the $\theta$ phase in the medial PFC entrains $\gamma$ power of the lateral PFC in a similar time window as the interregional phase synchronization effects (Fig. $7 H-L$ ). This entrainment could reflect the transmission of information from the DMPFC to the DLPFC during excitable phases of DMPFC $\theta$ oscillations and may thus be a result of the preceding predominant $\mathrm{DMPFC} \rightarrow$ DLPFC $\theta$ coupling direction.

Similar to phase synchronization, this CFC effect was also more pronounced for correct compared with incorrect trials. These results show that DMPFC and DLPFC communicate via phase synchronization in the $\theta$ band and via $\theta$-to- $\gamma$ coupling when a conflict is encountered. Furthermore, this interaction is important for the resolution of conflict. To our knowledge, this is the first direct evidence that modulation of prefrontal activity by the phase of $\theta$ oscillations in another region plays a functional role in humans.

In addition to their role in conflict resolution, DMPFCDLPFC interactions seem to also mediate conflict adaptation. Enhanced $\theta$ phase synchronization and enhanced $\theta$-to- $\gamma$ coupling in conflict trials reoccurred after responses were given. The latter effect, however, predicted faster reaction times for conflicts in the subsequent trial, suggestive of preparatory processes for the next trial. Results from the Granger analysis indicate that the directionality of communication between the two regions reversed after responses were given. In the second half of the trial, $\theta$ activity in the DMFC was driven by the DLPFC (Fig. 8).

What function could such a feedback signal serve? Previous studies have shown that DMPFC activity is decreased when a conflict has been preceded by another conflict, compared with a nonconflict trial (Botvinick et al., 1999, Kerns et al., 2004). This is thought to reflect decreased levels of detected conflict (and faster reaction times) because of enhanced cognitive control (Gratton et al., 1992). However, decreased conflict does not result from altered stimulus properties, but from adaptations in stimulusresponse mappings, diminishing response conflict. Thus, for a region to be able to constantly monitor conflicts between different response tendencies, it needs to be kept up-to-date with current response strategies and therefore requires information from areas involved in the implementation of the appropriate behavior (conflict resolution). We suggest that the postresponse DLPFC $\rightarrow$ DMPFC $\theta$ information flow and increased $\gamma$ to $\theta$ coupling reflect the neural signature of such an internal updating signal.

\section{Proposed model}

Together, our results draw, for the first time, a detailed picture of the neural dynamics underlying conflict detection, resolution, and adaptation and allow us to define the time frames and behavioral relevance of each of these subprocesses (Fig. 9). First, excitability within the DLPFC increases (Fig. $4 E, F$ ) in a temporally structured manner (organized at $\theta$ frequency; Fig. $7 E-G$ ). This effect starts immediately after stimulus onset and ends at already $\sim 330$ ms (i.e., $\sim 300 \mathrm{~ms}$ before any interactions with the DMPFC). Intraregional CFC may thus reflect a preparatory process even before sensory information is available. The detection 
of conflict starts at $\sim 290 \mathrm{~ms}$ after stimulus presentation, reflected by an increase in DMPFC $\theta$ power (Fig. $4 A-D$ ). After a short delay, DMPFC-DLPFC $\theta$ synchronization increases $(640-712$ ms; Fig. $7 A-D$ ), and the asymmetric preresponse results from GC analyses indicate that the increased interactions are actually driven by the DMPFC (Fig. 8). Briefly afterward (at 725 ms; Fig. $7 H-L)$, the conflict signal induces an alignment of DLPFC $\gamma$-band activity to the phase of DMPFC $\theta$ oscillations and enables successful conflict resolution. After the response, the interaction occurs in the opposite direction: Again, $\theta$ phase synchronization increases (in several time windows starting at $1480 \mathrm{~ms}$; Fig. 7A$D$ ). Concomitantly (at $1719 \mathrm{~ms}$; Fig. $7 \mathrm{H}-\mathrm{L}$ ), DLPFC $\gamma$ power is again locked to the phase of DMPFC $\theta$ oscillations, but now GC analyses indicate that interactions are driven by the DLPFC (Fig. 8). In brief, our data show that conflict detection, control, and adaptation are supported by a sequence of processes that use the interplay of $\theta$ and $\gamma$ oscillations in and between the DMPFC and DLPFC.

\section{References}

Axmacher N, Mormann F, Fernández G, Cohen MX, Elger CE, Fell J (2007) Sustained neural activity patterns during working memory in the human medial temporal lobe. J Neurosci 27:7807-7816. CrossRef Medline

Axmacher N, Henseler MM, Jensen O, Weinreich I, Elger CE, Fell J (2010) Cross-frequency coupling supports multi-item working memory in the human hippocampus. Proc Natl Acad Sci US A 107:3228-3233. CrossRef Medline

Barrett AB, Murphy M, Bruno MA, Noirhomme Q, Boly M, Laureys S, Seth AK (2012) Granger causality analysis of steady-state electroencephalographic signals during propofol-induced anaesthesia. PLoS One 7:e29072. CrossRef Medline

Bollimunta A, Chen Y, Schroeder CE, Ding M (2008) Neuronal mechanisms of cortical alpha oscillations in awake-behaving macaques. J Neurosci 28:9976-9988. CrossRef Medline

Botvinick MM, Braver TS, Barch DM, Carter CS, Cohen JD (2001) Conflict monitoring and cognitive control. Psych Rev 108:624-652. CrossRef Medline

Botvinick MM, Cohen JD, Carter CS (2004) Conflict monitoring and anterior cingulate cortex: an update. Trends Cogn Sci 8:539-546. CrossRef Medline

Botvinick M, Nystrom LE, Fissell K, Carter CS, Cohen JD (1999) Conflict monitoring versus selection-for-action in anterior cingulate cortex. Nature 402:179-181. CrossRef Medline

Brovelli A, Ding M, Ledberg A, Chen Y, Nakamura R, Bressler SL (2004) Beta oscillations in a large-scale sensorimotor cortical network: directional influences revealed by Granger causality. Proc Natl Acad Sci U S A 101:9849-9854. CrossRef Medline

Bruhn P, Parsons OA (1977) Reaction time variability in epileptic and brain-damaged patients. Cortex 13:373-384. CrossRef Medline

Buzsáki G (2006) Rhythms of the brain. Oxford: Oxford UP.

Buzsáki G, Draguhn A (2004) Neuronal oscillations in cortical networks. Science 304:1926-1929. CrossRef Medline

Canolty RT, Knight RT (2010) The functional role of cross-frequency coupling. Trends Cogn Sci 14:506-515. CrossRef Medline

Canolty RT, Edwards E, Dalal SS, Soltani M, Nagarajan SS, Kirsch HE, Berger MS, Barbaro NM, Knight RT (2006) High $\gamma$ power is phase-locked to $\theta$ oscillations in human neocortex. Science 313:1626-1628. CrossRef Medline

Cavanagh JF, Cohen MX, Allen JJ (2009) Prelude to and resolution of an error: EEG phase synchrony reveals cognitive control dynamics during action monitoring. J Neurosci 29:98-105. CrossRef Medline

Cavanagh JF, Zambrano-Vazquez L, Allen JJ (2012) Theta lingua franca: a common mid-frontal substrate for action monitoring processes. Psychophysiology 49:220-238. CrossRef Medline

Cohen MX, Ridderinkhof KR, Haupt S, Elger CE, Fell J (2008) Medial frontal cortex and response conflict: evidence from human intracranial EEG and medial frontal cortex lesion. Brain Res 1238:127-142. CrossRef Medline

Coste CP, Sadaghiani S, Friston KJ, Kleinschmidt A (2011) Ongoing brain activity fluctuations directly account for intertrial and indirectly for in- tersubject variability in Stroop Task Performance. Cereb Cortex 21:26122619. CrossRef Medline

Crone NE, Miglioretti DL, Gordon B, Lesser RP (1998) Functional mapping of human sensorimotor cortex with electrocorticographic spectral analysis: II. Event-related synchronization in the $\gamma$ band. Brain 121:2301-2315. CrossRef Medline

Cui J, Xu L, Bressler SL, Ding M, Liang H (2008) BSMART: a Matlab/C toolbox for analysis of multichannel neural time series. Neural Netw 21: 1094-1104. CrossRef Medline

Ding M, Bressler SL, Yang W, Liang H (2000) Short-window spectral analysis of cortical event-related potentials by adaptive multivariate autoregressive modelling: data preprocessing, model validation, and variability assessment. Biol Cybern 83:35-45. CrossRef Medline

Fell J, Axmacher N (2011) The role of phase synchronization in memory processes. Nat Rev Neurosci 12:105-118. CrossRef Medline

Fell J, Klaver P, Lehnertz K, Grunwald T, Schaller C, Elger CE, Fernández G (2001) Human memory formation is accompanied by rhinalhippocampal coupling and decoupling. Nat Neurosci 4:1259-1264. CrossRef Medline

Fries P (2005) A mechanism for cognitive dynamics: neuronal communication through neuronal coherence. Trends Cogn Sci 9:474-480. CrossRef Medline

Gratton G, Coles MG, Donchin E (1992) Optimizing the use of information: strategic control of activation of responses. J Exp Psychol Gen 121: 480-506. CrossRef Medline

Hanslmayr S, Pastötter B, Bäuml K, Gruber S, Wimber M, Klimesch W (2008) The electrophysiological dynamics of interference during the Stroop Task. Cognitive Neurosci 20:215-225. CrossRef Medline

Haupt S, Axmacher N, Cohen MX, Elger CE, Fell J (2009) Activation of the caudal anterior cingulate cortex due to task-related interference in an auditory Stroop paradigm. Hum Brain Mapp 30:3043-3056. CrossRef Medline

Huynh H, Feldt LS (1976) Estimation of the box correction for degrees of freedom from sample data in randomized block and split-plot designs. J Educ Behav Stat 1:69-82. CrossRef

Jensen O, Colgin LL (2007) Cross-frequency coupling between neuronal oscillations. Trends Cogn Sci 11:267-269. CrossRef Medline

Jensen O, Kaiser J, Lachaux JP (2007) Human $\gamma$-frequency oscillations associated with attention and memory. Trends Neurosci 30:317-324. CrossRef Medline

Jurkiewicz MT, Gaetz WC, Bostan AC, Cheyne D (2006) Post-movement beta rebound is generated in motor cortex: evidence from neuromagnetic recordings. Neuroimage 32:1281-1289. CrossRef Medline

Kerns JG, Cohen JD, MacDonald AW 3rd, Cho RY, Stenger VA, Carter CS (2004) Anterior cingulate conflict monitoring and adjustments in control. Science 303:1023-1026. CrossRef Medline

Koga S, Rothermel R, Juhász C, Nagasawa T, Sood S, Asano E (2011) Electrocorticographic correlates of cognitive control in a Stroop task-intracranial recording in epileptic patients. Hum Brain Mapp 32:1580-1591. CrossRef Medline

Lachaux JP, Rodriguez E, Martinerie J, Varela FJ (1999) Measuring phase synchrony in brain signals. Hum Brain Mapp 8:194-208. CrossRef Medline

Lisman J (2005) The $\theta / \gamma$ discrete phase code occurring during the hippocampal phase precession may be a more general brain coding scheme. Hippocampus 15:913-922. CrossRef Medline

MacDonald AW 3rd, Cohen JD, Stenger VA, Carter CS (2000) Dissociating the role of the dorsolateral prefrontal and the anterior cingulate cortex in cognitive control. Science 288:1835-1838. CrossRef Medline

MacLeod CM (1991) Half a century on the Stroop effect: an integrative review. Psychol Bull 109:163-203. CrossRef Medline

Mansouri FA, Buckley MJ, Tanaka K (2007) Mnemonic function of the dorsolateral prefrontal cortex in conflict-induced behavioral adjustment. Science 318:987-990. CrossRef Medline

Mansouri FA, Tanaka K, Buckley MJ (2009) Conflict-induced behavioural adjustment: a clue to the executive functions of the prefrontal cortex. Nat Rev Neurosci 10:141-152. CrossRef Medline

Maris E, Oostenveld R (2007) Nonparametric statistical testing of EEG- and MEG-data. J Neurosci Methods 164:177-190. CrossRef Medline

Mayr U, Awh E, Laurey P (2003) Conflict adaptation effects in the absence of executive control. Nat Neurosci 6:450-452. CrossRef Medline

Michels L, Bucher K, Lüchinger R, Klaver P, Martin E, Jeanmonod D, Bran- 
deis D (2010) Simultaneous EEG-fMRI during a working memory task: modulations in low and high frequency bands. PLoS One 5:e10298. CrossRef Medline

Miller KJ, Leuthardt EC, Schalk G, Rao RP, Anderson NR, Moran DW, Miller JW, Ojemann JG (2007) Spectral changes in cortical surface potentials during motor movement. J Neurosci 27:2424-2432. CrossRef Medline

Mitchell WG, Zhou Y, Chavez JM, Guzman BL (1992) Reaction time, attention, and impulsivity in epilepsy. Pediatr Neurol 8:19-24. CrossRef Medline

Morishima Y, Okuda J, Sakai K (2010) Reactive mechanism of cognitive control system. Cereb Cortex 20:2675-2683. CrossRef Medline

Nunez PL, Srinivasan R (2006) Electric fields of the brain: the neurophysics of EEG, 2nd Ed. New York: Oxford UP.

Oostenveld R, Fries P, Maris E, Schoffelen JM (2011) FieldTrip: open source software for advanced analysis of MEG, EEG, and invasive electrophysiological data. Comput Intell Neurosci 2011:156869. CrossRef Medline

Pastötter B, Berchtold F, Bäuml KH (2012) Oscillatory correlates of controlled speed-accuracy tradeoff in a response-conflict task. Hum Brain Mapp 33:1834-1849. CrossRef Medline

Pfurtscheller G, Lopes da Silva FH (1999) Event-related EEG/MEG synchronization and desynchronization: basic principles. Clin Neurophysiol 110:1842-1857. CrossRef Medline

Ridderinkhof KR, Ullsperger M, Crone EA, Nieuwenhuis S (2004) The role of the medial frontal cortex in cognitive control. Science 306:443-447. CrossRef Medline

Seth AK (2010) A MATLAB toolbox for Granger causal connectivity analysis. J Neurosci Methods 186:262-273. CrossRef Medline

Siegel M, Donner TH, Engel AK (2012) Spectral fingerprints of large-scale neuronal interactions. Nat Rev Neurosci 13:121-134. CrossRef Medline

Staudigl T, Hanslmayr S, Bäuml KH (2010) Theta oscillations reflect the dynamics of interference in episodic memory retrieval. J Neurosci 30: 11356-11362. CrossRef Medline

Stern ER, Mangels JA (2006) An electrophysiological investigation of preparatory attentional control in a spatial Stroop task. J Cogn Neurosci 18:1004-1017. CrossRef Medline

Stroop JR (1935) Studies of interference in serial verbal reactions. J Exp Psychol 18:643-662. CrossRef

Swann NC, Tandon N, Pieters TA, Aron AR (2013) Intracranial electroencephalography reveals different temporal profiles for dorsal- and ventrolateral prefrontal cortex in preparing to stop action. Cereb Cortex 23: 2479-2488. CrossRef Medline

Trujillo LT, Allen JJ (2007) Theta EEG dynamics of the error-related negativity. Clin Neurophysiol 118:645-668. CrossRef Medline

van Veen V, Carter CS (2005) Separating semantic conflict and response conflict in the Stroop task: a functional MRI study. Neuroimage 27:497504. CrossRef Medline

Varela F, Lachaux JP, Rodriguez E, Martinerie J (2001) The brainweb: phase synchronization and large-scale integration. Nat Rev Neurosci 2:229_ 239. CrossRef Medline

von Stein A, Sarnthein J (2000) Different frequencies for different scales of cortical integration: from local $\gamma$ to long range alpha/ $\theta$ synchronization. Int J Psychophysiol 38:301-313. CrossRef Medline

Wang C, Ulbert I, Schomer DL, Marinkovic K, Halgren E (2005) Responses of human anterior cingulate cortex microdomains to error detection, conflict monitoring, stimulus-response mapping, familiarity, and orienting. J Neurosci 25:604-613. CrossRef Medline

Ward LM (2003) Synchronous neural oscillations and cognitive processes. Trends Cogn Sci 7:553-559. CrossRef Medline

Womelsdorf T, Johnston K, Vinck M, Everling S (2010) Theta-activity in anterior cingulate cortex predicts task rules and their adjustments following errors. Proc Natl Acad Sci U S A 107:5248-5253. CrossRef Medline 